ACS Catalysis

Supporting Information for:

\title{
Reactivity of Hydrogen On and In Nanostructured Molybdenum Nitride: Crotonaldehyde Hydrogenation
}

\author{
Brian M. Wyvratt, ${ }^{a}$ Jason R. Gaudet, ${ }^{a}$ Daniel B. Pardue, ${ }^{b}$ Andrea Marton, ${ }^{b}$ \\ Svemir Rudic, ${ }^{d}$ Elizabeth A. Mader, *c Thomas R. Cundari, *b James M. Mayer ${ }^{* c}$, \\ Levi T. Thompson *a
}
${ }^{a}$ Department of Chemical Engineering and Hydrogen Energy Technology Laboratory, University of Michigan, Ann Arbor, MI 48109-2136, USA
${ }^{b}$ Department of Chemistry, Center for Advanced Scientific Computing and Modeling (CASCaM), University of North Texas, Denton, TX, 76203, USA
${ }^{c}$ Department of Chemistry, Yale University, PO Box 208107 New Haven, CT, 06520, USA
${ }^{d}$ ISIS Pulsed Neutron Facility, STFC, Rutherford Appleton Laboratory, Chilton, Didcot, Oxfordshire, OX11 0QX, UK

Corresponding Authors

* E-mail: 1tt@umich.edu (L.T.T.)

* E-mail: james.mayer@yale.edu (J.M.M)

*E-mail: t@unt.edu (T.R.C.)

* E-mail: elizabeth.mader@yale.edu (E.A.M.) 
X-ray Diffraction data

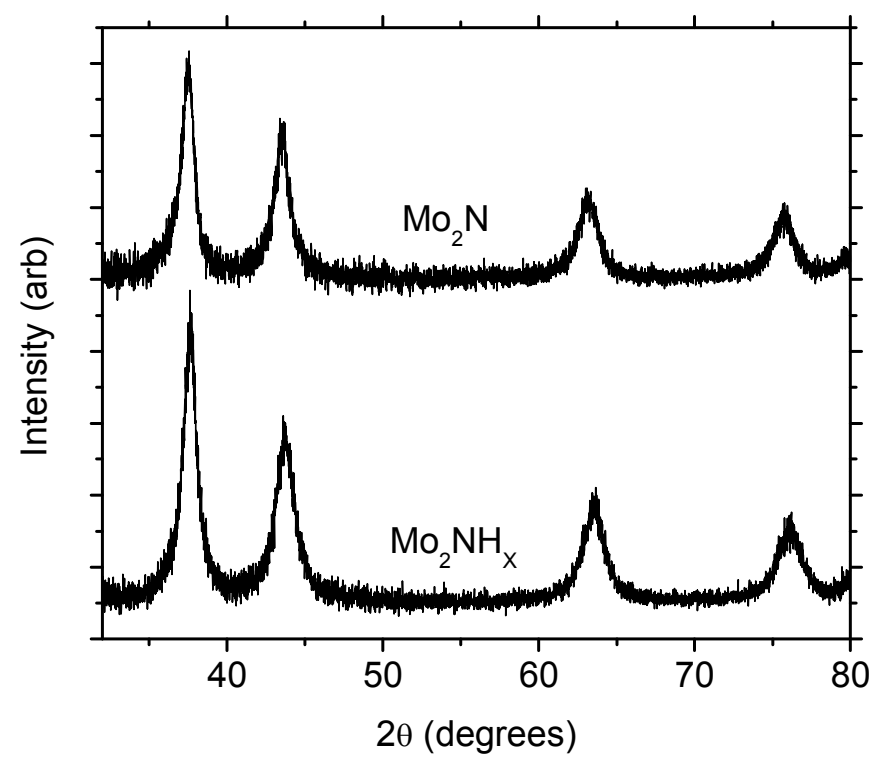

Figure S1. X-ray diffraction pattern for $\gamma-\mathrm{Mo}_{2} \mathrm{~N}$ prior to hydrogen treatment and after hydrogen treatment.

Table S1. Properties of $\gamma-\mathrm{Mo}_{2} \mathrm{~N}$ and $\gamma-\mathrm{Mo}_{2} \mathrm{NH}_{\mathrm{x}}$ catalysts

\begin{tabular}{ccccc}
\hline & $\begin{array}{c}\text { BET Surface } \\
\text { area }\left(\mathbf{m}^{\mathbf{2}} \mathbf{g}^{-\mathbf{1}}\right)\end{array}$ & $\begin{array}{c}\text { ICDD } \\
\text { PDF\# }\end{array}$ & $\begin{array}{c}\text { Crystallite } \\
\text { size }(\mathbf{n m})\end{array}$ & $\begin{array}{c}\text { Unit cell length } \\
\text { parameter }(\mathbf{n m})\end{array}$ \\
\hline$\gamma-\mathrm{Mo}_{2} \mathrm{~N}$ & $103.0 \pm 0.3$ & $00-025-1366$ & $7.9 \pm 1.2$ & $0.4180 \pm 0.001$ \\
$\gamma-\mathrm{Mo}_{2} \mathrm{NH}_{\mathrm{x}}$ & $103 \pm 0.2$ & $00-025-1366$ & $6.9 \pm 1.3$ & $0.4142 \pm 0.001$ \\
\hline
\end{tabular}

Crotonaldehyde Hydrogenation Measurements.

For the crotonaldhyde experiments, larger scale samples of $\gamma-\mathrm{Mo}_{2} \mathrm{~N}-\mathrm{H}_{\mathrm{x}}$ were prepared using the hydrogen addition conditions described in $\S 2.3$, with the following adjustments. Approximately $950 \mathrm{mg}$ of the passivated $\gamma-\mathrm{Mo}_{2} \mathrm{~N}$ was used per batch. Multiple $\gamma-\mathrm{Mo}_{2} \mathrm{~N}$ batches were synthesized to minimize batch-to-batch performance differences. The flow rates of the $10 \% \mathrm{H}_{2} / \mathrm{Ar}$ were adjusted to $665 \mathrm{~mL} \mathrm{~min}^{-1}$ in order to maintain comparable 
gas hourly space velocity with the larger weight of catalyst. The heating rates, times, and gas concentrations were unchanged.

Gas Chromatography Analysis for Crotonaldehyde Hydrogenation

GC-FID analysis was performed using a Varian CP-3800 equipped with a Poropak Q $(0.53 \mu \mathrm{m}$ diameter, $25 \mathrm{~m}$ length $)$ and argon carrier gas. The injector temperature was set at $220^{\circ} \mathrm{C}$ and the column temperature profile was as follows: $60^{\circ} \mathrm{C}$ for $10 \mathrm{~min}$, heat to $180^{\circ} \mathrm{C}$ at $15^{\circ} \mathrm{C} \mathrm{min}^{-1}$, heat to $220^{\circ} \mathrm{C}$ at $10^{\circ} \mathrm{C} \min ^{-1}$, then hold at this temperature for 8 minutes. GC-MS analysis was performed using a Shimadzu QP-2010 GC-MS equipped with a DB-5 column (30m, $0.25 \mathrm{~mm}$ ID). The injector temperature was set to $250^{\circ} \mathrm{C}$ and the column was heated from $75^{\circ} \mathrm{C}$ to $250^{\circ} \mathrm{C}$ at $5^{\circ} \mathrm{C} \mathrm{min}^{-1}$.

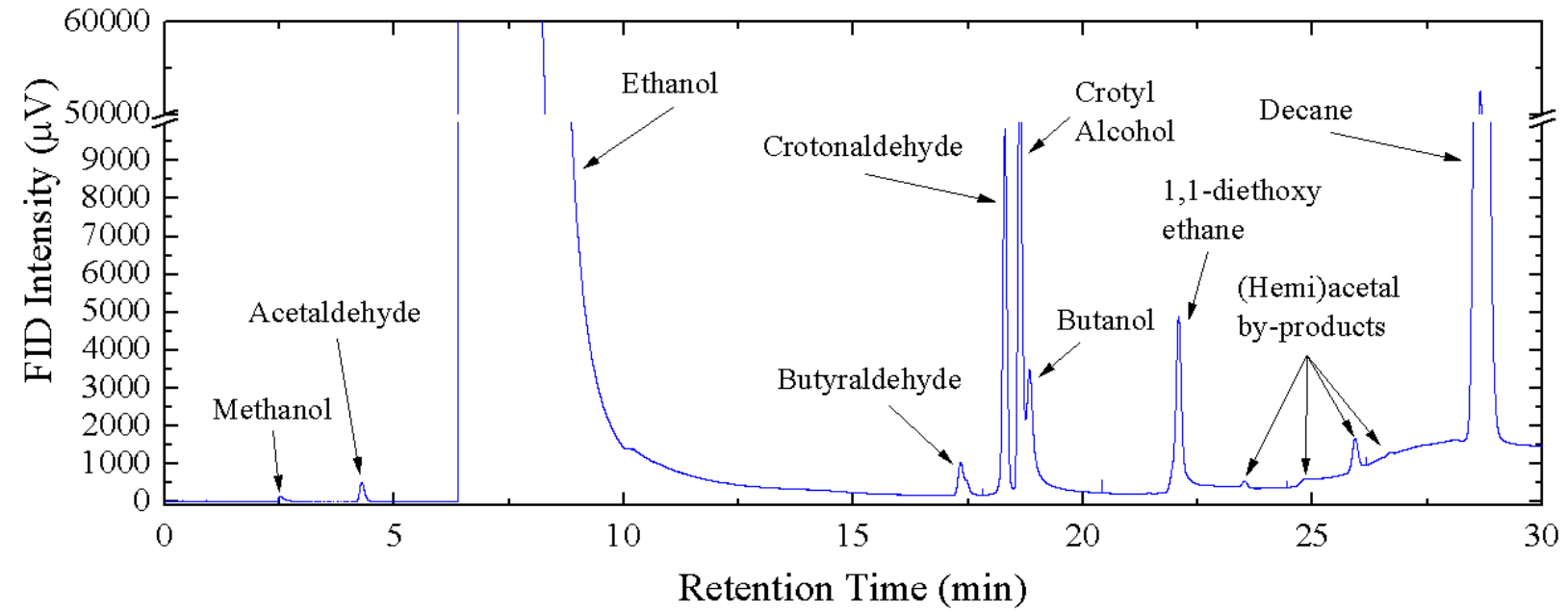

Figure S2. Prototypical GC trace with retention times of products. Note: 1,1-diethoxy ethane and acetaldehyde formed from ethanol solvent.

CA Pulse Chemisorption Experiments

CA pulse chemisorption was performed on a "purged" $\gamma-\mathrm{Mo}_{2} \mathrm{~N}$ surface using a Micromeritics Autochem 2920 system equipped with a thermal conductivity detector and 
a Pfeiffer Vacuum Quadstar GSD-301 Mass Spectrometer. Prior to the pulse chemisorption, approximately $100 \mathrm{mg}$ of the catalysts were treated in an identical manner to that described in Section 2.3 of the manuscript to produce the "purged" surface. The reactor was then heated to $35^{\circ} \mathrm{C}$ and the material was exposed to pulses of crotonaldehyde $(\sim 0.72 \% \mathrm{CA} / \mathrm{He}$ a $500 \mu \mathrm{L}$ loop $)$ while monitoring the effluent gas using both TCD and MS detectors. CA was pulsed until the surface became saturated as indicated by the constant peak area in the TCD towards the latter part of the experiment, and the uptake was quantified relative to the saturation peak area. The CA uptake for a "purged" $\gamma-\mathrm{Mo}_{2} \mathrm{~N}$ surface was calculated to be $\sim 190 \mu \mathrm{mol} \mathrm{g}{ }^{-1}$ or 1.1 sites $\mathrm{nm}^{-2}$.

Thermal Desorption Data

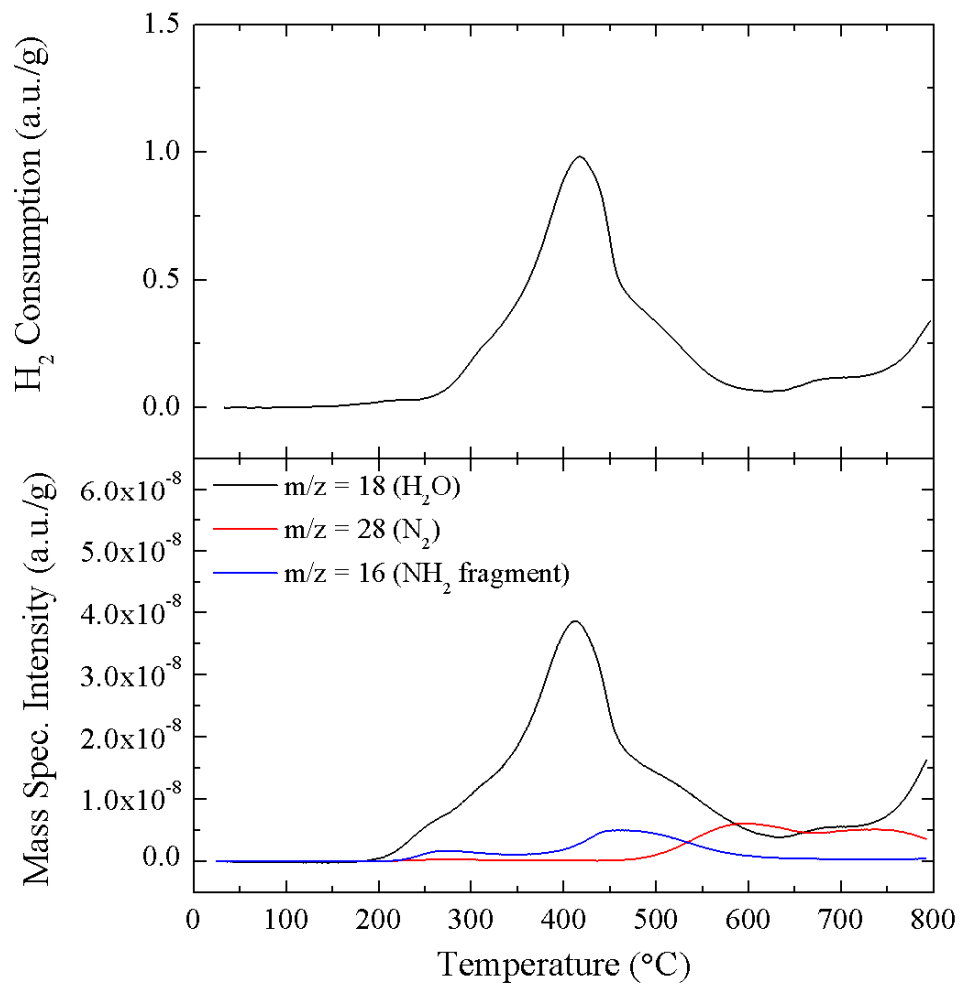

Figure S3. $\mathrm{H}_{2}$ Temperature-programmed reduction of passivated $\gamma-\mathrm{Mo}_{2} \mathrm{~N}$, showing signals for the thermal conductivity detector (top) and mass spectrometer (bottom). 


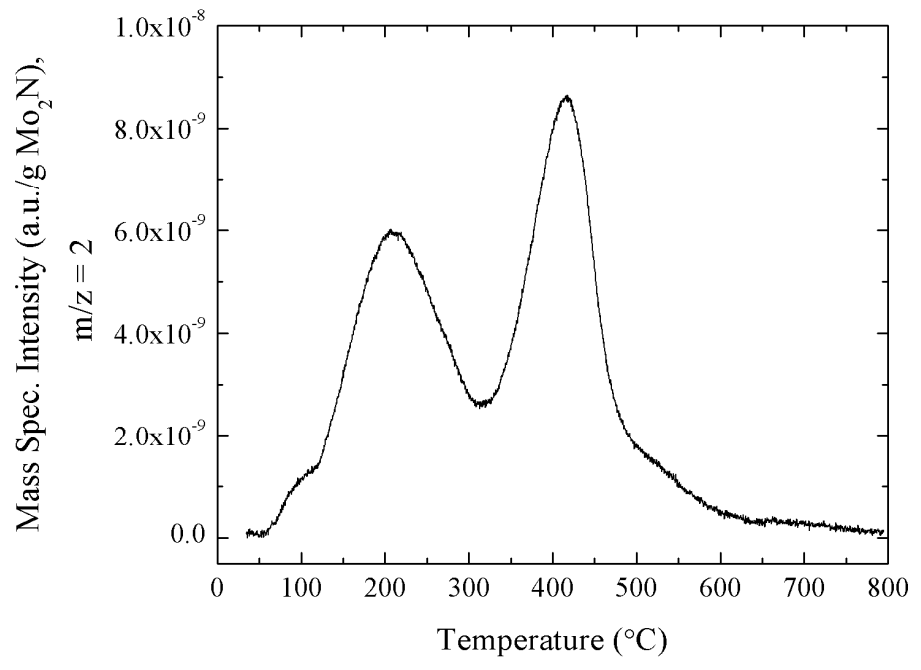

Figure S4. Thermal-desorption spectra for hydrogen from $\gamma-\mathrm{Mo}_{2} \mathrm{~N}$ following hydrogen reduction at $500^{\circ} \mathrm{C}$ for $0.5 \mathrm{hr}$.

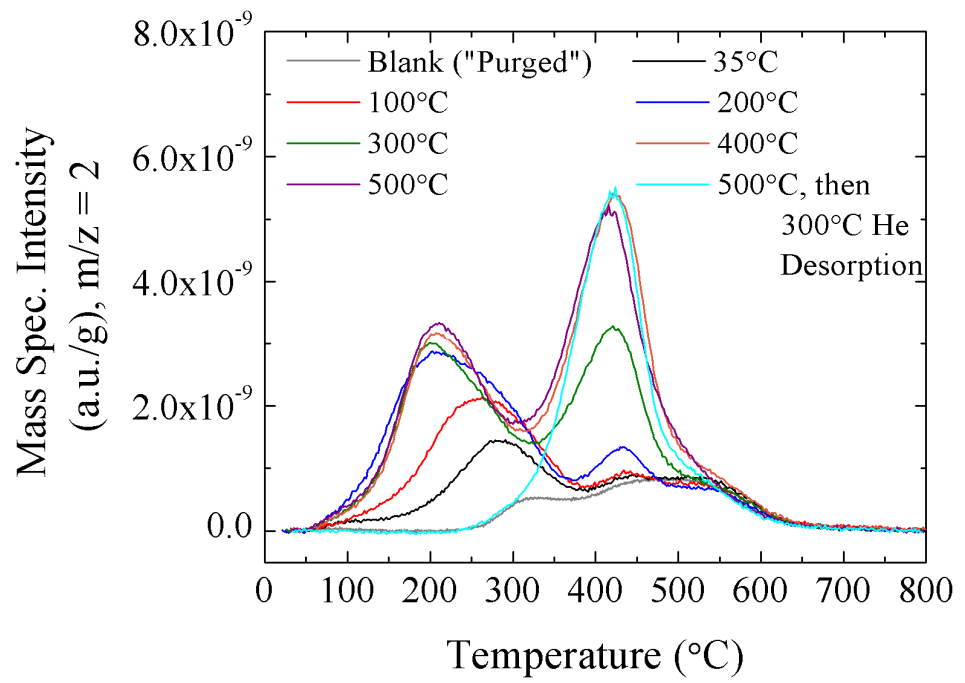

Figure S5. Thermal desorption spectra illustrating the hydrogen distribution in $\gamma-\mathrm{Mo}_{2} \mathrm{~N}-\mathrm{H}_{\mathrm{x}}$ following exposure to $10 \% \mathrm{H}_{2} / \mathrm{Ar}$ for $0.5 \mathrm{hr}$ at different temperatures. All spectra were taken following $\mathrm{H}_{2}$ loading after a reduction and "surface purging" desorption step as described in the experimental section. Heating rate of $15^{\circ} \mathrm{C} \mathrm{min}^{-1}$. Note: the "Blank 'Purged"" sample still shows 2 desorption peaks $\left(\sim 300^{\circ} \mathrm{C}\right.$ and $\left.\sim 500^{\circ} \mathrm{C}\right)$, attributed to Peak A and Peak B at low coverages. 


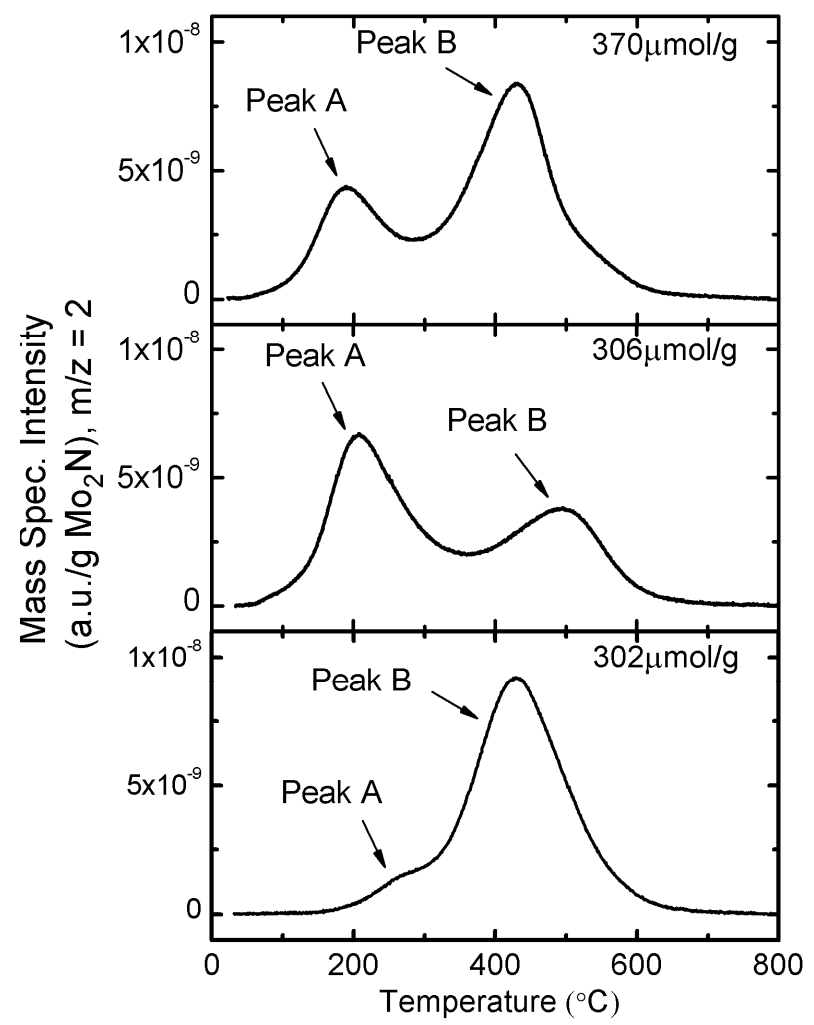

Figure S6. Thermal desorption spectra of hydrogen in $\gamma-\mathrm{Mo}_{2} \mathrm{~N}-\mathrm{H}_{\mathrm{x}}$ following selective hydrogen population. Heating rate $15^{\circ} \mathrm{C} \mathrm{min}^{-1}$. Total amount of $\mathrm{H}_{2}$ desorbed shown in $\mu \mathrm{mol} \mathrm{g}{ }^{-1}$. The top, middle, and bottom panels represent the $\mathrm{H}_{2}$ desorption spectra following treatment at $500^{\circ} \mathrm{C}$ in $10 \% \mathrm{H}_{2} / \mathrm{Ar}$ for $0.5 \mathrm{~h}, 200^{\circ} \mathrm{C}$ in $10 \% \mathrm{H}_{2} / \mathrm{Ar}$ for $0.5 \mathrm{~h}$, and $500^{\circ} \mathrm{C}$ in $10 \% \mathrm{H}_{2} / \mathrm{Ar}$ for $0.5 \mathrm{~h}$ followed by $300^{\circ} \mathrm{C}$ in $\mathrm{He}$ for $0.5 \mathrm{~h}$, respectively. 


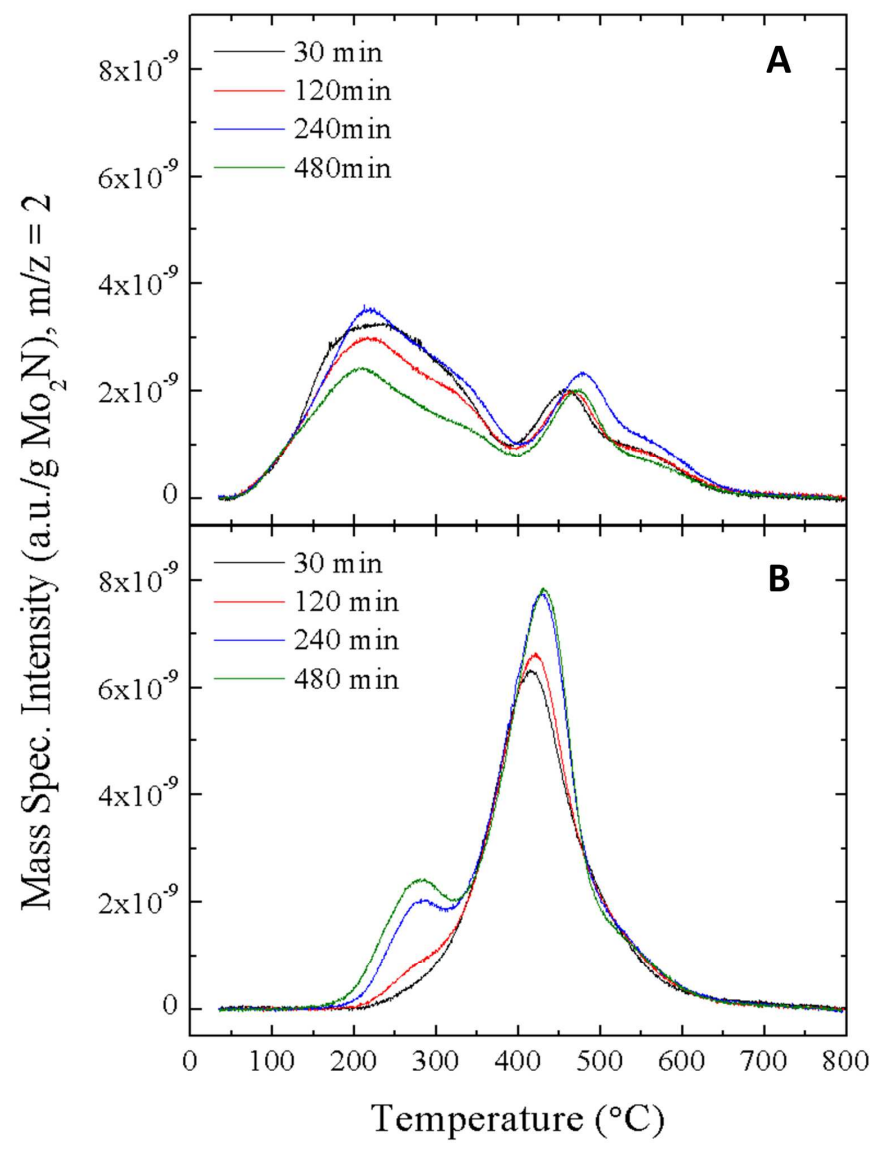

Figure S7. Thermal desorption spectra of hydrogen from $\gamma-\mathrm{Mo}_{2} \mathrm{~N}$ following hydrogen treatment at (a) $200{ }^{\circ} \mathrm{C}$ for $0.5 \mathrm{hr}$ (site A) and (b) $500{ }^{\circ} \mathrm{C}$ for $0.5 \mathrm{hr}$, followed by desorption in $\mathrm{He}$ at $300^{\circ} \mathrm{C}$ for $0.5 \mathrm{hr}$ (site B) aimed at monitoring diffusion between sites. The spectra represent the following "aging" times in $\mathrm{He}\left(70 \mathrm{~mL} \mathrm{~min}^{-1}\right)$ at $35^{\circ} \mathrm{C}: 0.5,2,4$, $8 \mathrm{hr}$. Heating rate of $15^{\circ} \mathrm{C} \mathrm{min}^{-1}$. 


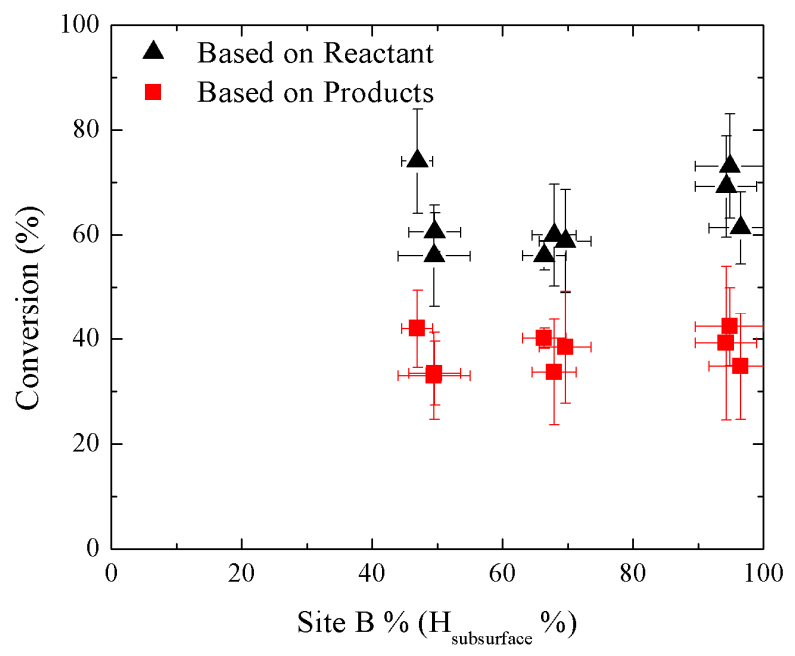

Figure S8. Conversions based on crotonaldehyde reactant and measured products for the $\gamma-\mathrm{Mo}_{2} \mathrm{~N}-\mathrm{H}_{\mathrm{x}} /$ Crotonaldehyde batch reactions. 
Inelastic Neutron Scattering Data

Table S2. Energies and Vibrational Frequencies of hydrogen addition to $\gamma-\mathrm{Mo}_{2} \mathrm{~N}$. (Energies are relative the energy minimized structure of $\gamma-\mathrm{Mo}_{2} \mathrm{~N}$ and the appropriate equivalents of $\mathrm{H}_{2}$ )

\begin{tabular}{|c|c|c|c|}
\hline $\begin{array}{c}\text { Model \& } \\
\text { Stoichiometry }\end{array}$ & $\begin{array}{l}\Delta E_{(\mathrm{H} \cdot \text { addn) }} \\
{\text { kcal } \text { mol }^{-1}}^{-1}\end{array}$ & $\begin{array}{c}\text { Dominant } \\
\text { Vibrations, } \mathbf{c m}^{-1}\end{array}$ & $\begin{array}{c}\text { Mode } \\
\text { Assignment }\end{array}$ \\
\hline$\gamma-\mathrm{Mo}_{2} \mathrm{~N}$ & 0 & $\begin{array}{l}612 \\
542 \\
523 \\
500 \\
470\end{array}$ & \\
\hline $\begin{array}{l}\kappa^{1}-\mathrm{NH}_{\text {surf }} \\
\gamma-\mathrm{Mo}_{2} \mathrm{NH}_{0.3}\end{array}$ & -6.6 & $\begin{array}{l}3328 \\
832 \\
800\end{array}$ & $\begin{array}{l}v\left(\mathrm{NH}_{\text {sym }}\right) \\
v\left(\mathrm{NH}_{\text {asym }}\right) \\
v\left(\mathrm{NH}_{\text {asym }}\right)\end{array}$ \\
\hline $\begin{array}{l}\mu^{6}-\mathrm{Mo}_{6} \mathrm{H}_{\text {sub }} \\
\gamma-\mathrm{Mo}_{2} \mathrm{NH}_{0.3}\end{array}$ & -6.4 & $\begin{array}{l}1324 \\
986 \\
652\end{array}$ & $\begin{array}{l}v(\text { asym }) \\
v(\text { sym }) \\
v(\text { asym })\end{array}$ \\
\hline $\begin{array}{l}\kappa^{1}-\mathrm{MoH}_{\text {surf }} \\
\gamma-\mathrm{Mo}_{2} \mathrm{NH}_{0.3}\end{array}$ & +6.3 & & \\
\hline $\begin{array}{l}\kappa^{1}-\mathrm{MoH}_{\text {surf }}+\kappa^{1}-\mathrm{NH}_{\text {surf }} \\
\gamma-\mathrm{Mo}_{2} \mathrm{NH}_{0.7}\end{array}$ & -1.4 & $\begin{array}{l}3372 \\
1742 \\
863\end{array}$ & $\begin{array}{l}v(\mathrm{NHasym}) \\
v\left(\mathrm{MoH}_{\text {sym }}\right) \\
v\left(\mathrm{NH}_{\mathrm{asym}}\right)\end{array}$ \\
\hline $\begin{array}{l}\mu^{6}-\mathrm{Mo}_{6} \mathrm{H}_{\text {sub }}+\kappa^{1}-\mathrm{NH}_{\text {surf }} \\
\gamma-\mathrm{Mo}_{2} \mathrm{NH}_{0.7}\end{array}$ & -13.3 & $\begin{array}{l}3332 \\
1325 \\
963 \\
831 \\
800\end{array}$ & $\begin{array}{l}v\left(\mathrm{NH}_{\text {sym }}\right) \\
v\left(\mu^{6}-\mathrm{H} \text { asym }\right) \\
v\left(\mu^{6}-\mathrm{H} \text { sym }\right) \\
v\left(\mathrm{NH}_{\text {asym }}\right) \\
v\left(\mathrm{NH}_{\text {asym }}\right)\end{array}$ \\
\hline $\begin{array}{l}\left(\kappa^{1}-\mathrm{NH}_{\text {surf }}\right)_{2} \\
\gamma-\mathrm{Mo}_{2} \mathrm{NH}_{0.7}\end{array}$ & -9.1 & & \\
\hline $\begin{array}{l}\mu^{2}-\mathrm{NH}_{2 \text {-surf }} \\
\gamma-\mathrm{Mo}_{2} \mathrm{NH}_{0.7}\end{array}$ & +0.5 & $\begin{array}{l}3483 \\
3383 \\
1496 \\
\end{array}$ & $\begin{array}{l}v\left(\mathrm{NH}_{2 \text { asym }}\right) \\
v\left(\mathrm{NH}_{2 \text { sym }}\right) \\
v\left(\mathrm{NH}_{2 \text { scissor }}\right)\end{array}$ \\
\hline
\end{tabular}


TSC15480-15486_140519B_n-Mo2NHx_17K-Summed

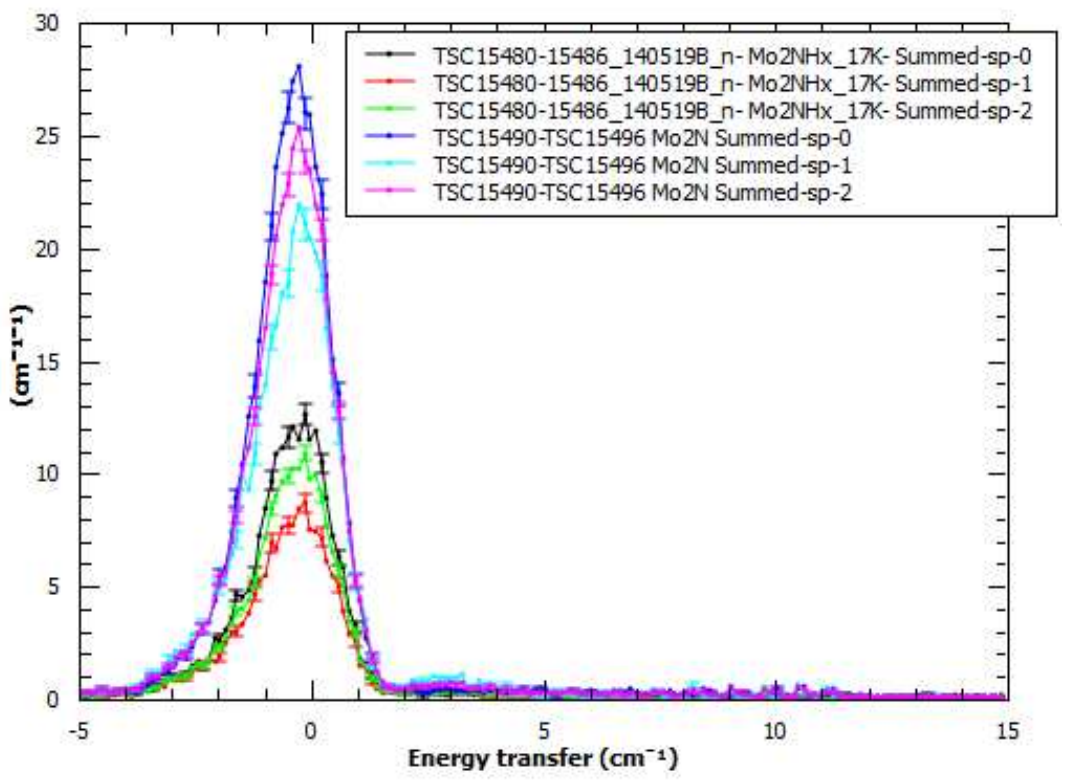

Figure S9: Elastic line for reduced $\gamma-\mathrm{Mo}_{2} \mathrm{~N}-\mathrm{H}_{\mathrm{x}}$ (forward detector, back detector, average) and "as prepared" $\gamma-\mathrm{Mo}_{2} \mathrm{~N}$ (forward detector, back detector, average).

TSC15490-TSC15496 Mo2N Summed

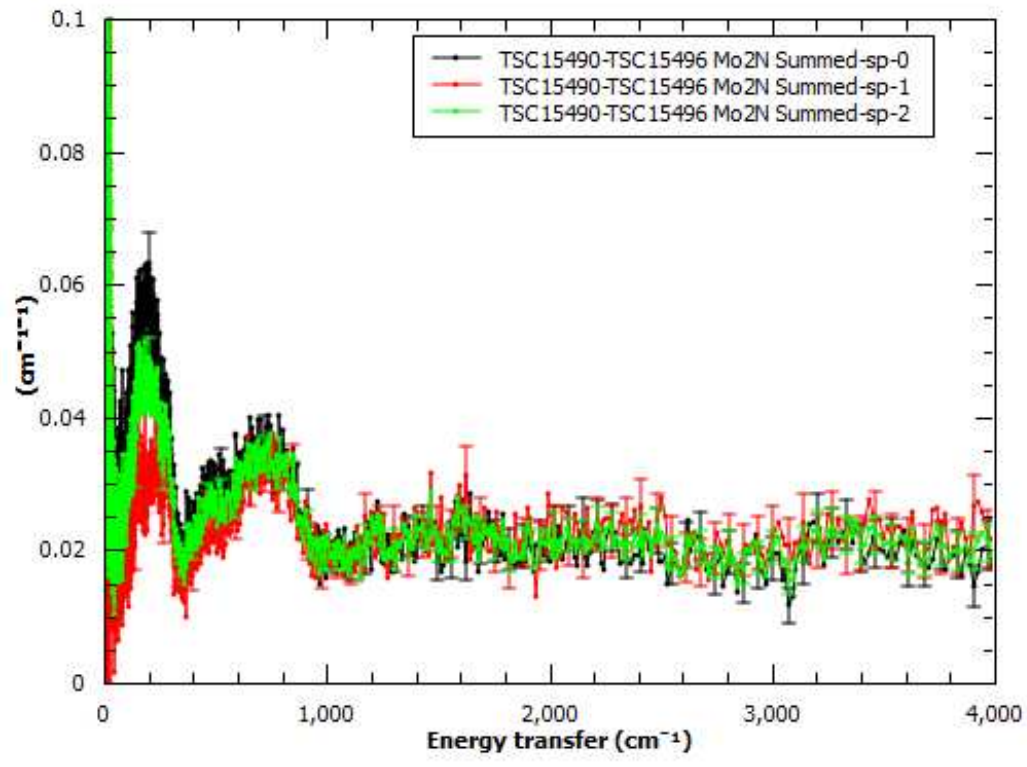

Figure S10: Raw inelastic scattering data for "as prepared" $\gamma-\mathrm{Mo}_{2} \mathrm{~N}$ (forward detector, back detector, average). 


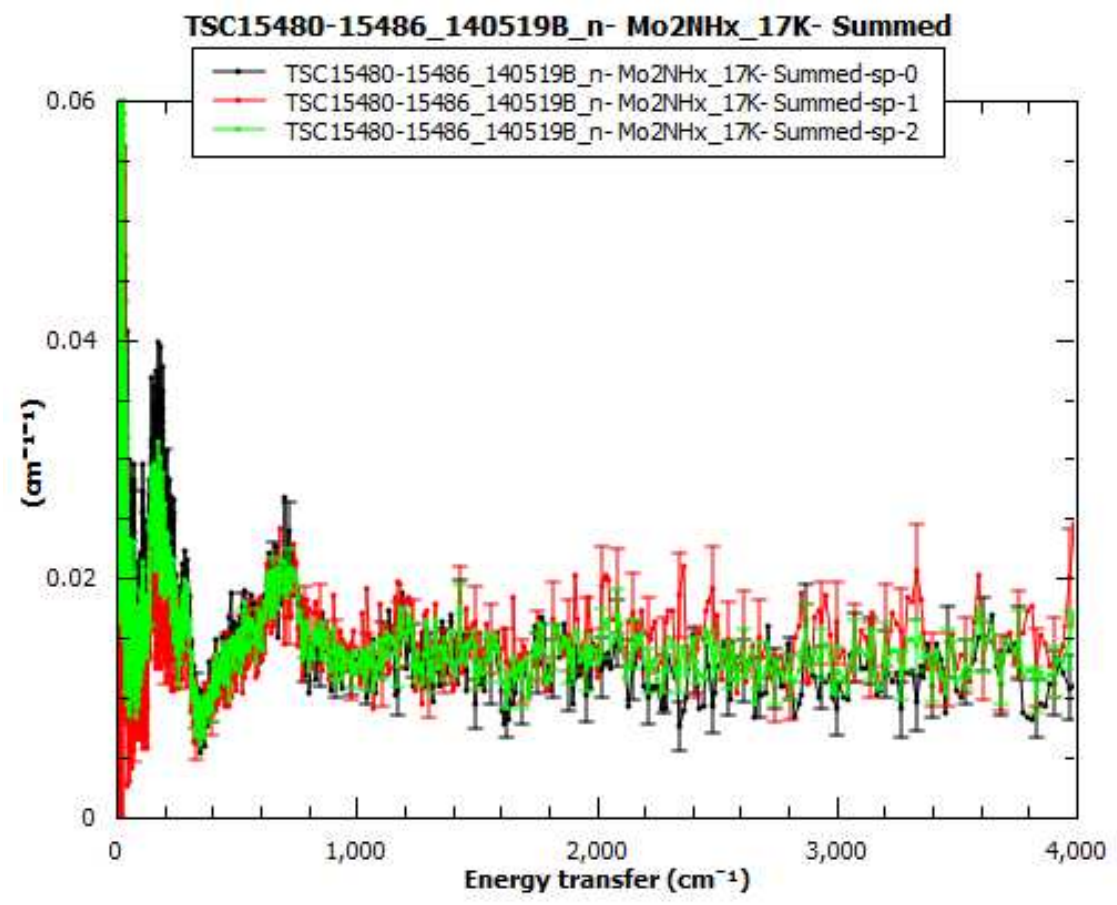

Figure S11: Raw inelastic scattering data for reduced $\gamma-\mathrm{Mo}_{2} \mathrm{~N}-\mathrm{H}_{\mathrm{x}}$ (forward detector, back detector, average).

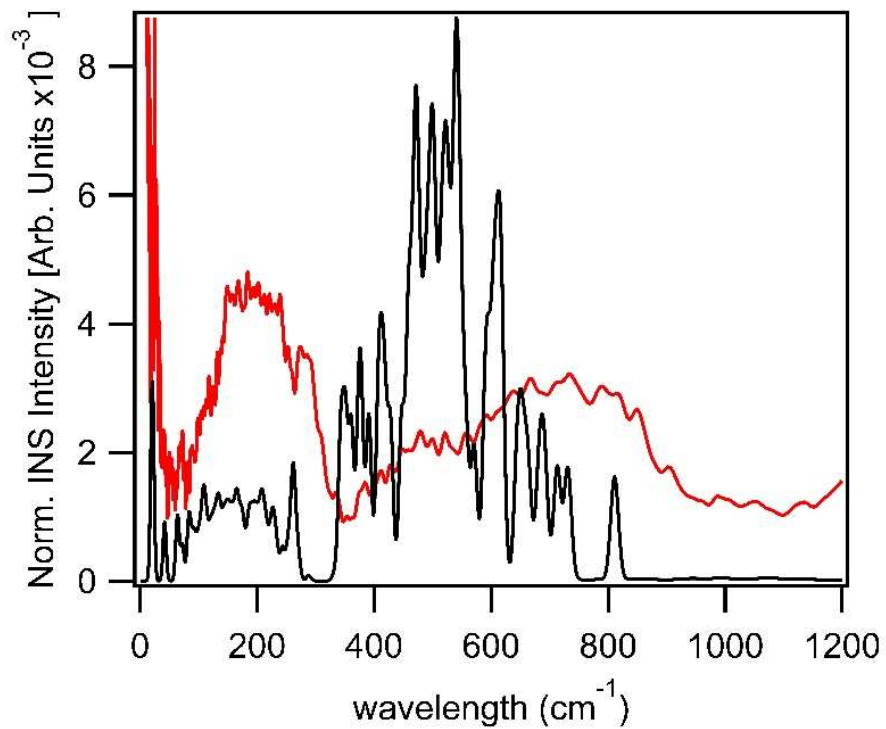

Figure S12: The low frequency region of the normalized $\gamma-\mathrm{Mo}_{2} \mathrm{~N}$ spectrum (red) compared to the simulated spectrum (black) from the VASP calculations below. 
A
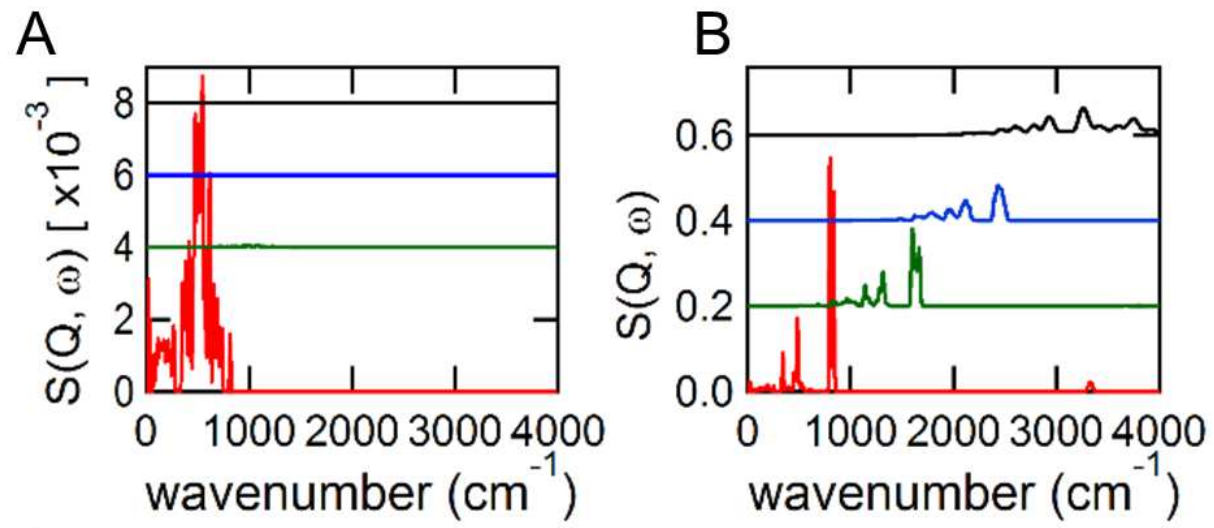

C
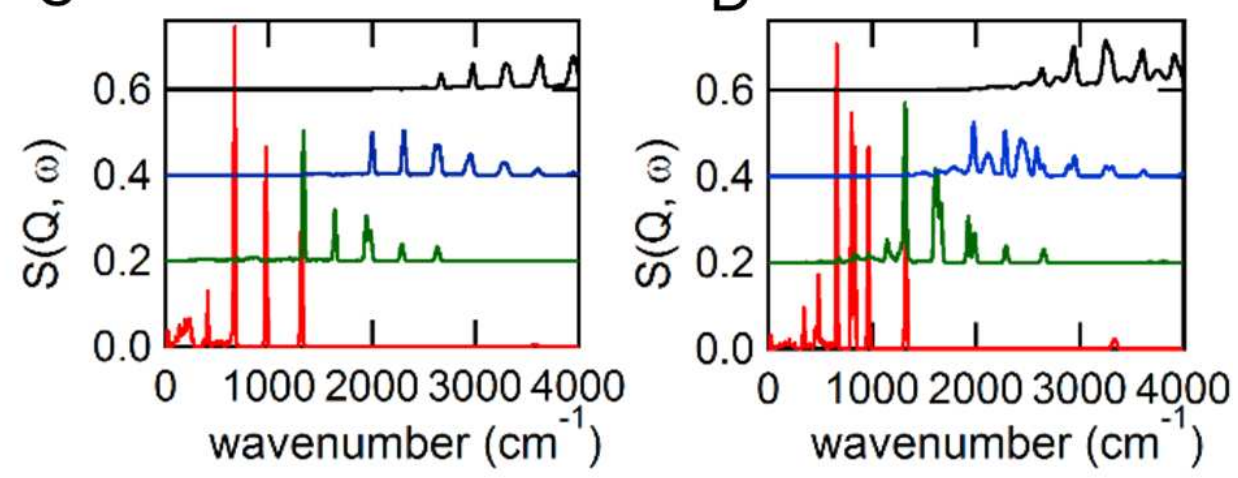

Figure S13. INS spectra simulated from VASP calculated phonon frequencies. Single (red) and multiple (2- green; 3- blue; 4- black) quantum number transitions are shown for four different structures. A) $\gamma-\mathrm{Mo}_{2} \mathrm{~N}$ B) $\gamma-\mathrm{Mo}_{2} \mathrm{NH}_{0.3}\left(\kappa^{1}-\mathrm{NH}_{\text {surf }}\right)$ C) $\gamma-\mathrm{Mo}_{2} \mathrm{NH}_{0.3}$ $\left(\mu^{6}-\mathrm{Mo}_{6} \mathrm{H}_{\text {sub }}\right)$ and D) $\gamma-\mathrm{Mo}_{2} \mathrm{NH}_{0.7}\left(\mu^{6}-\mathrm{Mo}_{6} \mathrm{H}_{\text {sub }}+\kappa^{1}-\mathrm{NH}_{\text {surf }}\right)$. 


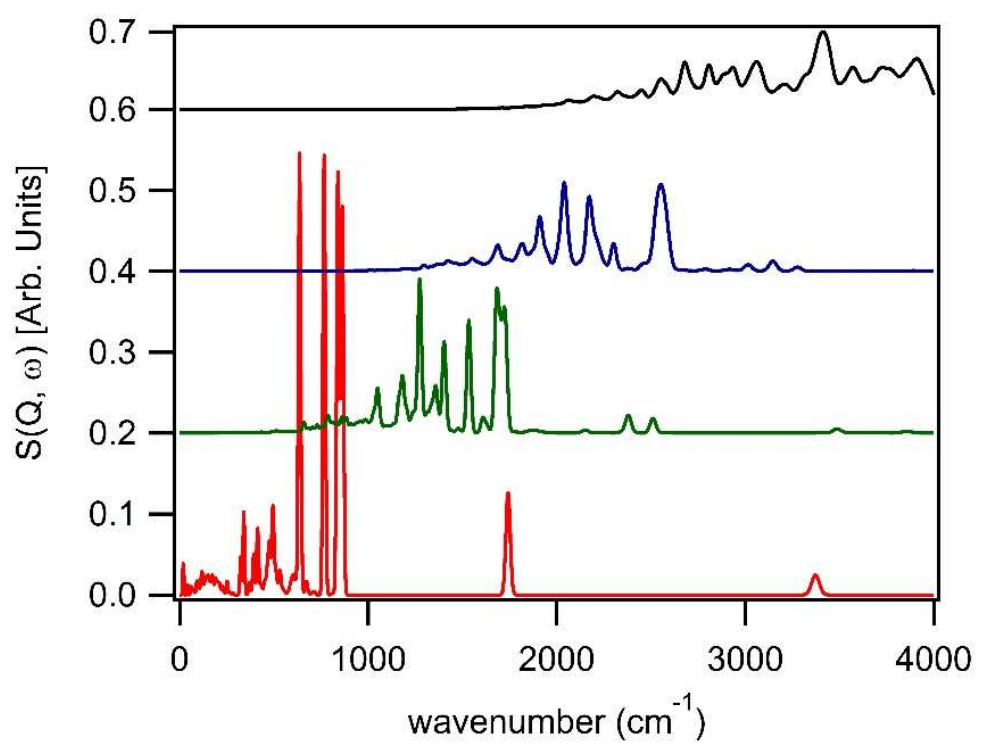

Figure S14: INS spectra simulated from VASP calculated phonon frequencies. Single (red) and multiple (2- green; 3- blue; 4- black) quantum number transitions are shown for 7 layer surface Mo-H with a surface $\mathrm{NH}$.

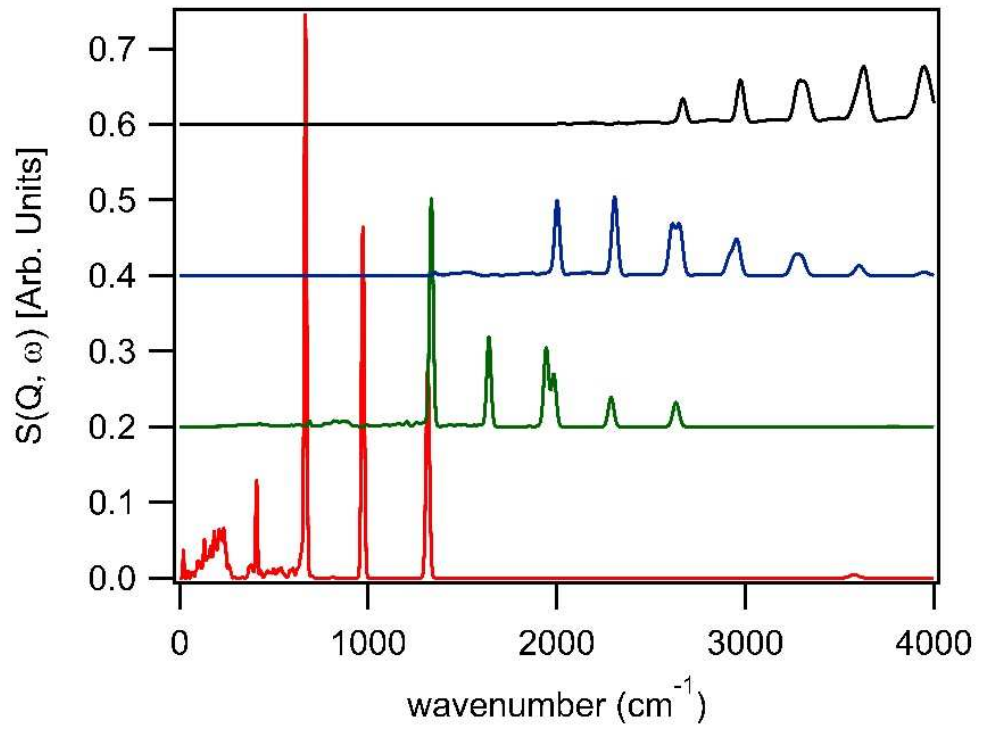

Figure S15: INS spectra simulated from VASP calculated phonon frequencies. Single (red) and multiple (2- green; 3- blue; 4- black) quantum number transitions are shown for 7-layer surface $\mathrm{OH}$. 


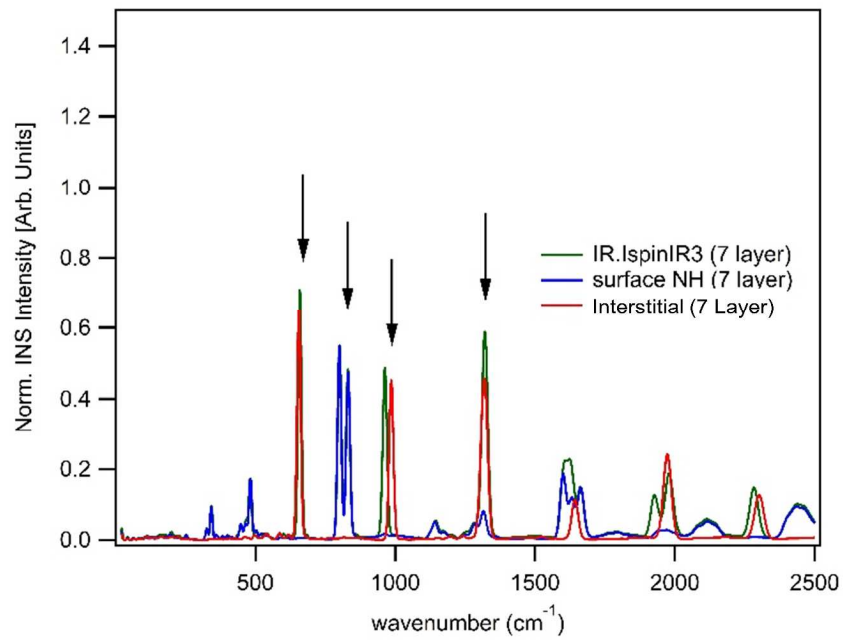

Figure S16: INS spectra simulated from VASP calculated phonon frequencies. The sum of single and multiple quantum number transitions are shown for $\gamma-\mathrm{Mo}_{2} \mathrm{NH}_{0.2}$-interstitial, $\gamma-\mathrm{Mo}_{2} \mathrm{NH}_{0.2^{-}}$(surface $\mathrm{NH}$ ), and $\gamma-\mathrm{Mo}_{2} \mathrm{NH}_{0.4^{-}}$(both surface $\mathrm{NH}$ and interstitial $\mathrm{H}$ ). The interstitial mode at $1000 \mathrm{~cm}^{-1}$ shifts in frequency when an $\mathrm{NH}$ group is added to the surface, suggesting the modes are coupled.

Details of the Computational Method

The tables below lists the coordinates and energies of the structures reported as they appear within the manuscript. Energies are reported in eV. Elements are reported by atomic number. Cartesian coordinates of the optimized atom position are reported in $\AA$.

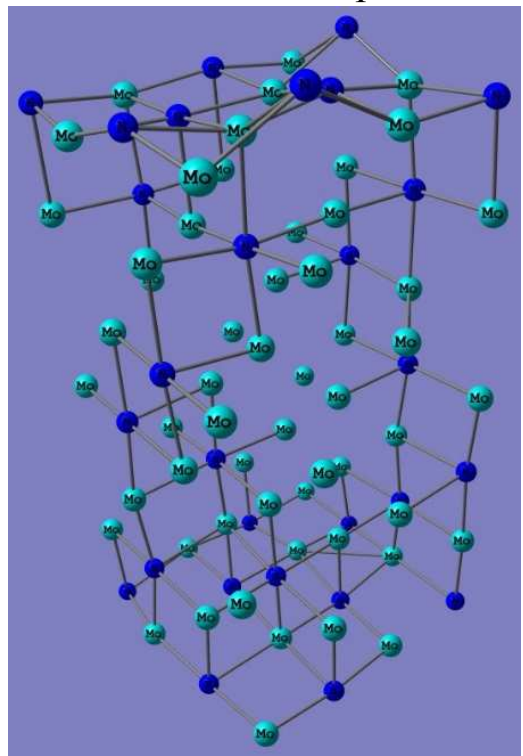

Figure S17: Image of energy minimized structure for $\gamma-\mathrm{Mo}_{2} \mathrm{~N}$. 


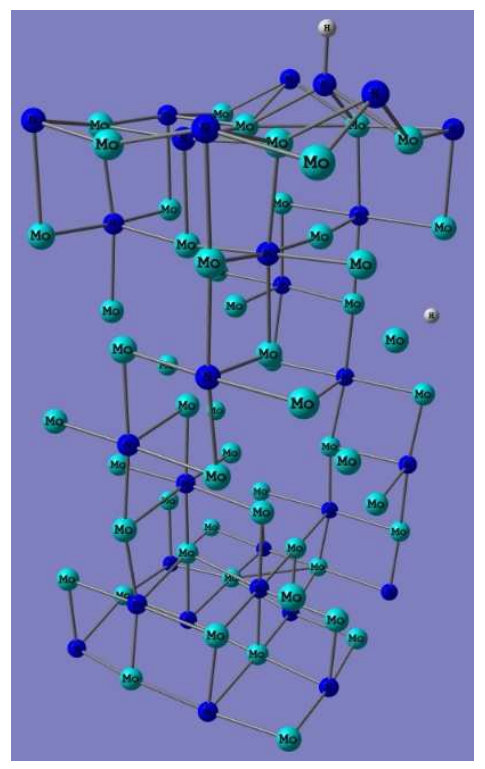

Figure S18: Image of energy minimized structure for $\gamma-\mathrm{Mo}_{2} \mathrm{NH}_{0.7}\left(\kappa^{1}-\mathrm{NH}, \mu^{6}-\mathrm{MoH}\right.$ Interstitial)

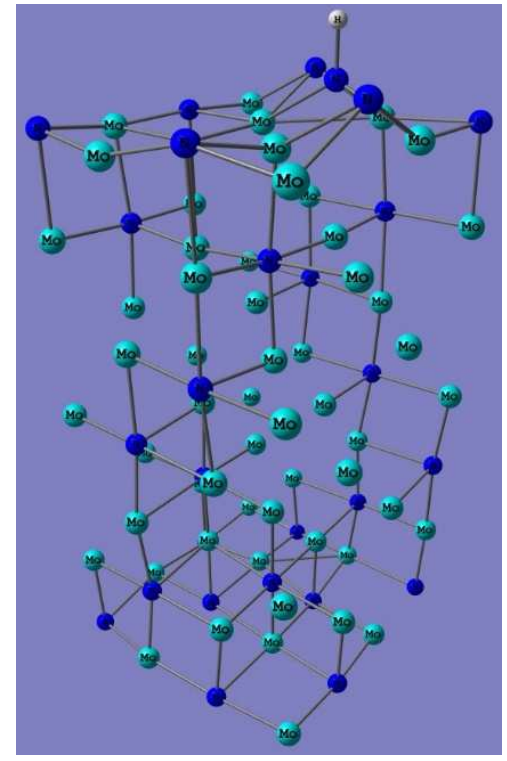

Figure S19: Image of energy minimized structure for $\gamma-\mathrm{Mo}_{2} \mathrm{NH}_{0.3}\left(\kappa^{1}-\mathrm{NH}\right)$ 


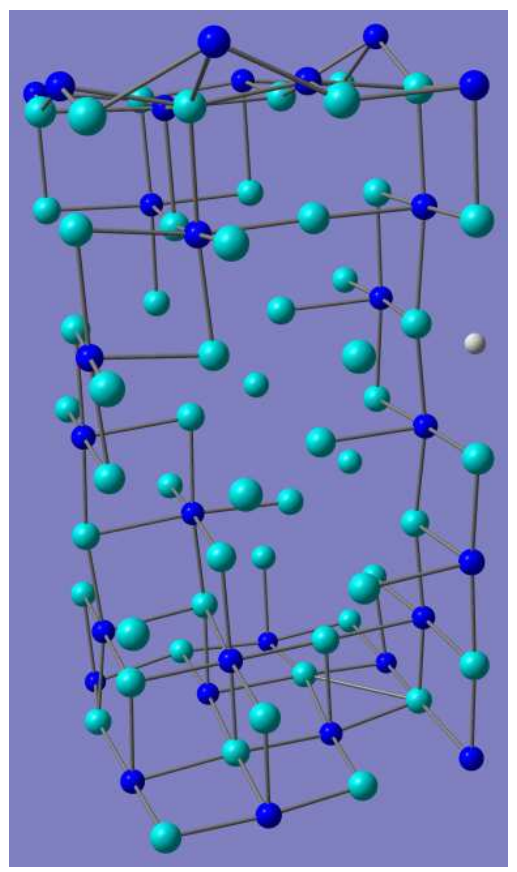

Figure S20: Image of energy minimized structure for $\gamma-\mathrm{Mo}_{2} \mathrm{NH}_{0.3}\left(\mu^{6}-\mathrm{MoH}\right.$ Interstitial)

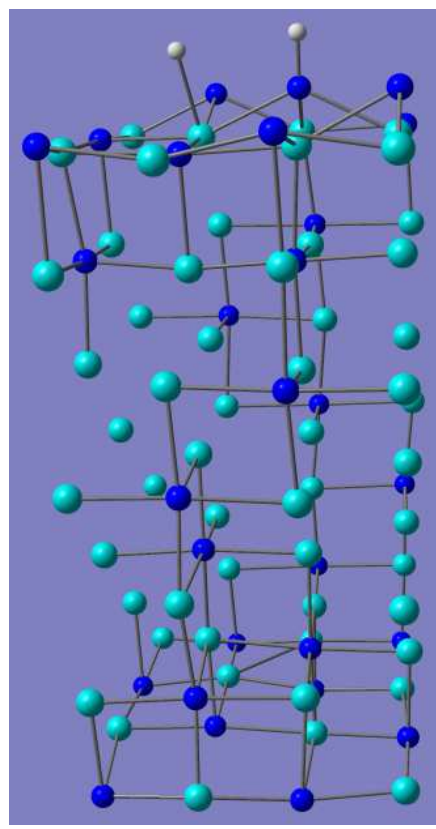

Figure S21: Image of energy minimized structure for $\gamma-\mathrm{Mo}_{2} \mathrm{NH}_{0.7}\left(\mu^{6}-\mathrm{MoH}, \kappa^{1}-\mathrm{NH}\right)$ 


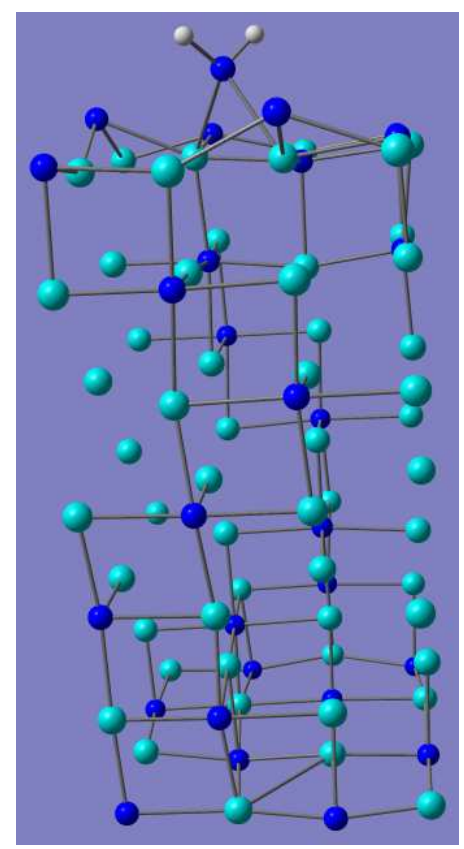

Figure S22: Image of energy minimized structure for $\gamma-\mathrm{Mo}_{2} \mathrm{NH}_{0.7}\left(\kappa^{1}-\mathrm{NH}_{2}\right)$

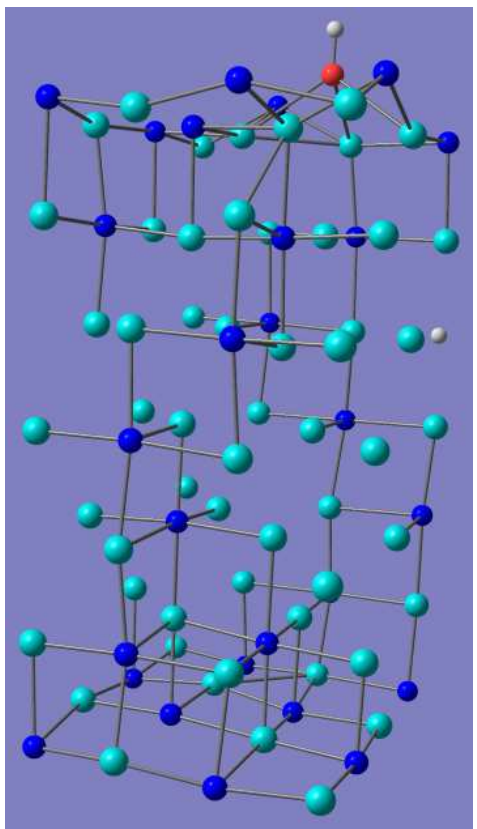

Figure S23: Image of energy minimized structure for $\gamma-\mathrm{Mo}_{2} \mathrm{~N}(\mathrm{OH})_{0.3}$ 


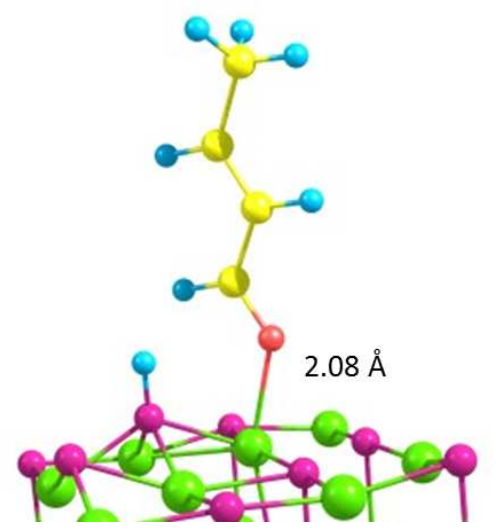

Figure S24: Image of energy minimized structure for $\kappa^{1}$-bound crotonaldehyde to $\gamma-\mathrm{Mo}_{2} \mathrm{~N}+2 \mathrm{H}$

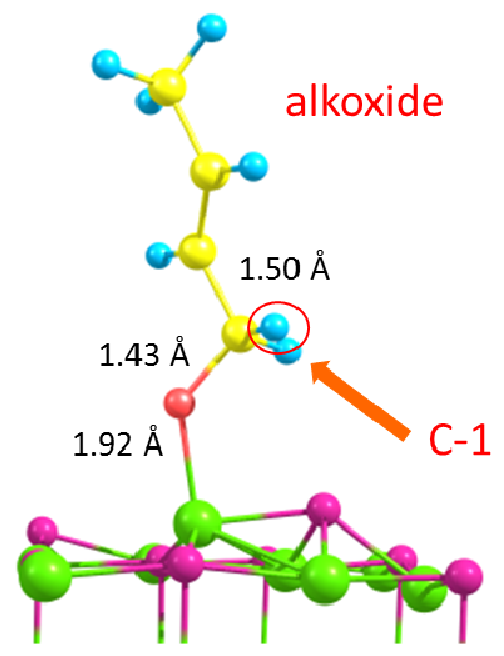

Figure S25: Image of energy minimized structure for "Alkoxide-bound to $\gamma-\mathrm{Mo}_{2} \mathrm{~N}+\mathrm{H}$ "

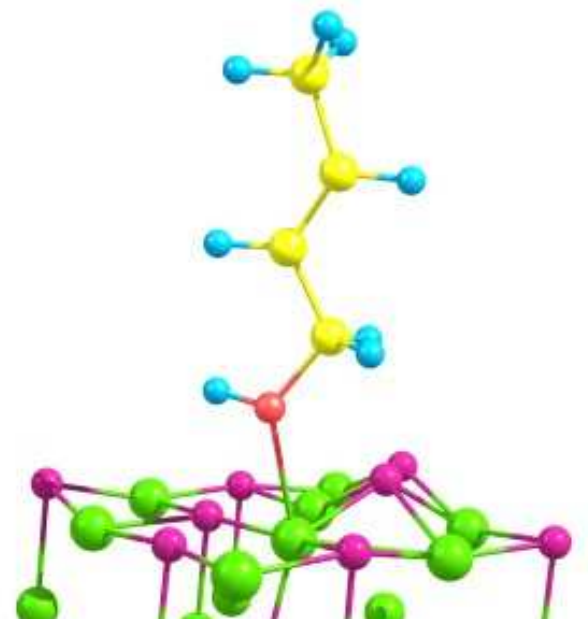

Figure S26: Image of energy minimized structure for " $\kappa^{1}-\mathrm{O}-$ crotyl alcohol-bound to $\gamma-\mathrm{Mo}_{2} \mathrm{~N} "$ 


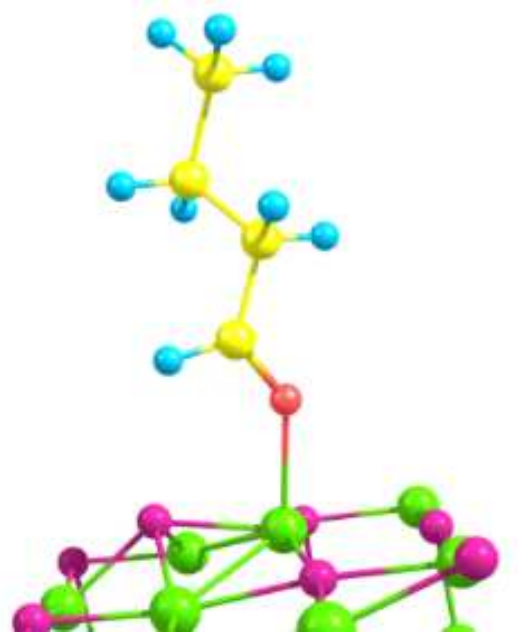

Figure S27: Image of energy minimized structure for "Butanal-bound to $\gamma-\mathrm{Mo}_{2} \mathrm{~N}$ "

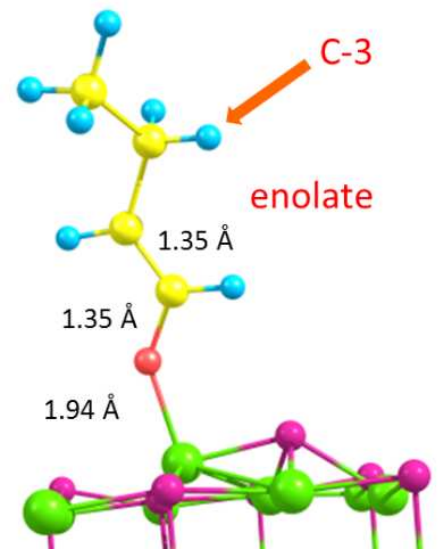

Figure S28: Image of energy minimized structure for "Enolate-bound to $\gamma-\mathrm{Mo}_{2} \mathrm{~N}+\mathrm{H}$ "

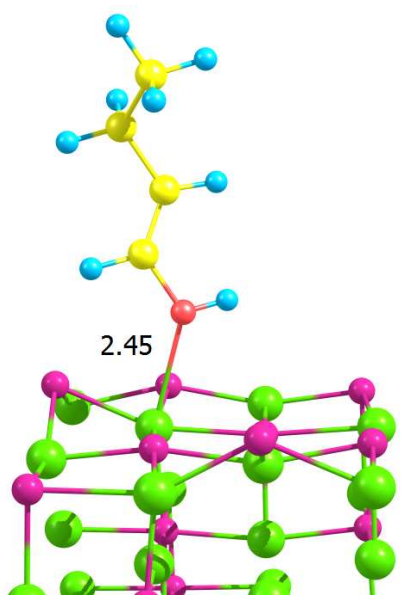

Figure S29: Image of energy minimized structure for "Enol-bound to $\gamma-\mathrm{Mo}_{2} \mathrm{~N}$ " 


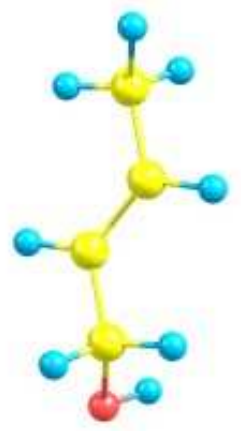

Figure S30: Image of Energy minimized structure for "CrotylAlcohol Alone"

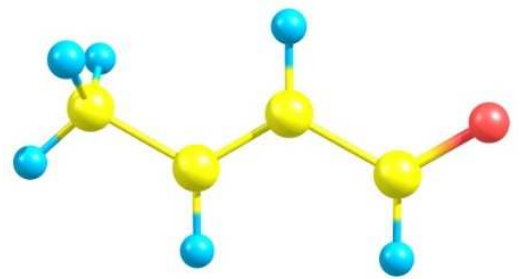

Figure S31: Image of Energy minimized structure for "Crotonaldehyde Alone"

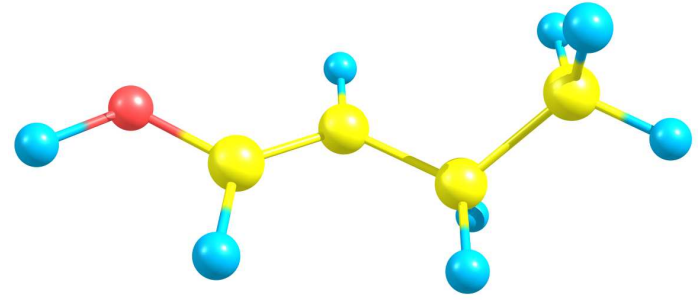

Figure S32: Image of Energy minimized structure for "Enol Alone"

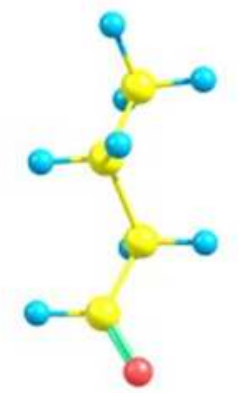

Figure S33: Image of Energy minimized structure for "Butanal Alone" 


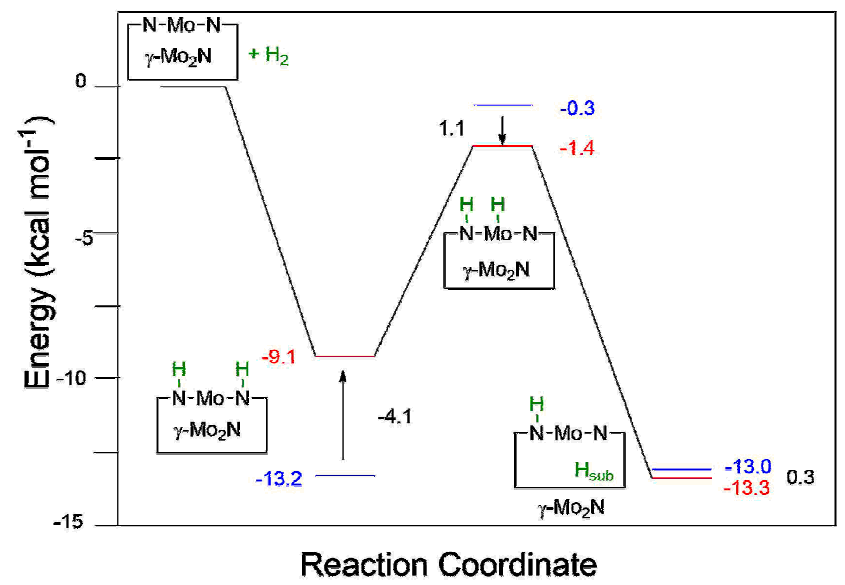

Figure S34. Estimates of ground-state thermodynamic cooperativity (black) calculated from a) the addition of two $\mathrm{H} \bullet$ per unit cell of $\gamma-\mathrm{Mo}_{2} \mathrm{~N}$ (red) compared to b) the sum of two $\gamma-\mathrm{Mo}_{2} \mathrm{~N}$ unit cells each containing one $\mathrm{H}^{\bullet}$ to (blue). 
Table S3: Coordinates and energy of $\gamma-\mathrm{Mo}_{2} \mathrm{~N}$ (Element Z, X, Y, Z)

$-846.568 \mathrm{eV}$

$\begin{array}{cccc}42 & 4.686840000 & 0.898030000 & 1.279610000 \\ 42 & 4.877240000 & 2.933740000 & 3.227770000 \\ 42 & 6.770330000 & 0.996760000 & 3.198520000 \\ 42 & 6.872680000 & 2.960430000 & 1.232570000 \\ 42 & 8.953010000 & 0.863170000 & 1.262700000 \\ 42 & 13.018290000 & 0.993060000 & 1.345160000 \\ 42 & 8.734860000 & 3.061600000 & 3.277340000 \\ 42 & 12.985000000 & 2.941050000 & 3.205450000 \\ 42 & 11.138540000 & 0.892180000 & 3.306920000 \\ 42 & 10.989990000 & 3.001780000 & 1.329450000 \\ 42 & 4.725050000 & 5.023950000 & 1.307980000 \\ 42 & 4.742170000 & 6.993670000 & 3.555530000 \\ 42 & 6.787970000 & 4.998440000 & 3.308530000 \\ 42 & 6.868490000 & 7.156480000 & 1.177260000 \\ 42 & 8.944240000 & 5.050270000 & 1.274100000 \\ 42 & 13.000900000 & 4.898310000 & 1.346740000 \\ 42 & 8.860930000 & 6.888590000 & 3.137910000 \\ 42 & 12.996070000 & 7.138520000 & 3.554210000 \\ 42 & 10.978690000 & 5.035770000 & 3.309630000 \\ 42 & 10.969180000 & 7.131680000 & 1.213360000 \\ 42 & 4.765480000 & 0.719550000 & 5.465540000 \\ 42 & 4.760370000 & 2.873210000 & 7.502610000 \\ 42 & 6.824020000 & 0.845730000 & 7.489830000 \\ 42 & 6.961880000 & 3.022870000 & 5.418700000 \\ 42 & 8.977230000 & 0.936680000 & 5.476560000 \\ 42 & 12.967960000 & 0.731660000 & 5.394080000 \\ 42 & 8.876740000 & 2.935340000 & 7.513960000 \\ 42 & 13.005580000 & 2.961660000 & 7.607060000 \\ 42 & 11.001130000 & 0.862470000 & 7.443150000 \\ 42 & 10.739080000 & 2.987400000 & 5.381910000 \\ 42 & 4.789450000 & 5.018030000 & 5.487090000 \\ 42 & 4.713780000 & 7.074570000 & 7.397830000 \\ 42 & 6.784990000 & 4.989850000 & 7.556870000 \\ 42 & 6.777930000 & 7.016340000 & 5.514720000 \\ 42 & 8.886940000 & 5.019900000 & 5.457410000 \\ 42 & 12.953800000 & 5.200140000 & 5.402230000 \\ 42 & 8.939420000 & 7.088500000 & 7.464010000 \\ 42 & 13.037020000 & 7.127760000 & 7.328660000 \\ 42 & 11.006890000 & 5.063310000 & 7.434750000 \\ 42 & 11.002220000 & 7.107540000 & 5.478650000 \\ 42 & 0.591450000 & 1.126840000 & 1.255520000 \\ 42 & 0.629250000 & 2.964470000 & 3.226190000\end{array}$

$\begin{array}{cccc}42 & 2.670460000 & 1.024670000 & 3.170950000 \\ 42 & 2.624040000 & 3.059860000 & 1.209940000 \\ 42 & 0.615420000 & 4.922290000 & 1.394410000 \\ 42 & 0.609530000 & 7.086800000 & 3.686500000 \\ 42 & 2.647010000 & 4.961390000 & 3.369420000 \\ 42 & 2.694740000 & 7.195570000 & 1.385590000 \\ 42 & 0.623110000 & 0.539070000 & 5.313640000 \\ 42 & 0.626920000 & 2.825120000 & 7.522320000 \\ 42 & 2.565820000 & 0.717150000 & 7.395340000 \\ 42 & 2.790610000 & 3.021090000 & 5.454420000 \\ 42 & 0.646740000 & 5.157730000 & 5.476410000 \\ 42 & 0.611110000 & 6.975120000 & 7.467160000 \\ 42 & 2.629240000 & 4.937120000 & 7.541560000 \\ 42 & 2.613180000 & 6.917230000 & 5.651790000 \\ 7 & 8.923680000 & 7.096510000 & 5.376640000 \\ 7 & 13.243060000 & 5.043900000 & 7.423010000 \\ 7 & 13.915910000 & 7.115440000 & 5.397860000 \\ 7 & 12.891440000 & 2.967940000 & 5.397100000 \\ 7 & 13.236020000 & 0.888430000 & 7.413740000 \\ 7 & 4.919210000 & 2.903880000 & 5.390500000 \\ 7 & 10.867720000 & 2.954080000 & 3.353440000 \\ 7 & 13.272940000 & 5.043230000 & 3.381530000 \\ 7 & 6.809850000 & 7.119750000 & 3.341690000 \\ 7 & 4.825360000 & 7.116550000 & 1.369830000 \\ 7 & 13.082220000 & 7.107080000 & 1.328380000 \\ 7 & 6.867120000 & 0.865940000 & 5.379510000 \\ 7 & 13.947690000 & 2.946100000 & 1.343050000 \\ 7 & 13.411590000 & 0.883920000 & 3.375880000 \\ 7 & 0.573670000 & 3.032140000 & 1.237590000 \\ 7 & 0.434740000 & 0.886920000 & 3.294200000 \\ 7 & 0.447030000 & 7.144750000 & 5.603730000 \\ 7 & 0.449000000 & 4.911220000 & 7.495910000 \\ 7 & 0.708950000 & 2.940440000 & 5.391740000 \\ 7 & 2.658680000 & 7.113210000 & 3.477920000 \\ 7 & 0.616260000 & 7.186680000 & 1.460770000 \\ 7 & 0.448360000 & 5.024660000 & 3.430810000 \\ 7 & 0.373870000 & 0.762070000 & 7.336510000 \\ 7 & 2.684290000 & 0.847970000 & 5.302240000 \\ 7 & 10.934120000 & 2.963550000 & 7.404930000 \\ 7 & 8.944060000 & 0.902900000 & 3.328650000 \\ 7 & 10.954360000 & 7.138320000 & 3.325450000 \\ 7 & 2.658730000 & 2.993030000 & 3.270190000\end{array}$


Table S4: Coordinates and energy of $\gamma-\mathrm{Mo}_{2} \mathrm{NH}_{0.7}\left(\kappa^{1}-\mathrm{NH}, \mu^{6}-\mathrm{MoH}\right.$ Interstitial), (Element Z, X, Y, Z)

$\begin{array}{cccc}-853.895 \mathrm{eV} & & \\ 42 & 4.684120000 & 0.893940000 & 1.276240000 \\ 42 & 4.867870000 & 2.940830000 & 3.242800000 \\ 42 & 6.754400000 & 1.010560000 & 3.195560000 \\ 42 & 6.849840000 & 2.948710000 & 1.244790000 \\ 42 & 8.957470000 & 0.862620000 & 1.267970000 \\ 42 & 13.020800000 & 0.997490000 & 1.356280000 \\ 42 & 8.742010000 & 3.027820000 & 3.280760000 \\ 42 & 12.996510000 & 2.972810000 & 3.233000000 \\ 42 & 11.139180000 & 0.885610000 & 3.308040000 \\ 42 & 10.992670000 & 2.982970000 & 1.334030000 \\ 42 & 4.727430000 & 5.013180000 & 1.302010000 \\ 42 & 4.738020000 & 6.984550000 & 3.559120000 \\ 42 & 6.821180000 & 5.004280000 & 3.323760000 \\ 42 & 6.858460000 & 7.146430000 & 1.209640000 \\ 42 & 8.904380000 & 5.012980000 & 1.279270000 \\ 42 & 13.039060000 & 4.908230000 & 1.429590000 \\ 42 & 8.865980000 & 6.950760000 & 3.219680000 \\ 42 & 13.023260000 & 7.032480000 & 3.529940000 \\ 42 & 10.867770000 & 5.034090000 & 3.276540000 \\ 42 & 10.989360000 & 7.116540000 & 1.236090000 \\ 42 & 4.763680000 & 0.724180000 & 5.471630000 \\ 42 & 4.765120000 & 2.881520000 & 7.513480000 \\ 42 & 6.834290000 & 0.835820000 & 7.489950000 \\ 42 & 6.961350000 & 3.024300000 & 5.421100000 \\ 42 & 8.977490000 & 0.956760000 & 5.481540000 \\ 42 & 12.977940000 & 0.692940000 & 5.388210000 \\ 42 & 8.875370000 & 2.913040000 & 7.523330000 \\ 42 & 12.994200000 & 2.947390000 & 7.631420000 \\ 42 & 11.020630000 & 0.854040000 & 7.448990000 \\ 42 & 10.744220000 & 3.001260000 & 5.384860000 \\ 42 & 4.794950000 & 5.021060000 & 5.495580000 \\ 42 & 4.718210000 & 7.069340000 & 7.397450000 \\ 42 & 6.820940000 & 5.003790000 & 7.552530000 \\ 42 & 6.765230000 & 7.028600000 & 5.515080000 \\ 42 & 8.864960000 & 5.018950000 & 5.446740000 \\ 42 & 12.997540000 & 5.151730000 & 5.355340000 \\ 42 & 8.934020000 & 7.114960000 & 7.407000000 \\ 42 & 13.018450000 & 7.098720000 & 7.309770000 \\ 42 & 10.923620000 & 5.087240000 & 7.455890000 \\ 42 & 11.022790000 & 7.098380000 & 5.469160000 \\ 42 & 0.591450000 & 1.126840000 & 1.255520000 \\ 42 & 0.629250000 & 2.964470000 & 3.226190000 \\ 42 & 2.670460000 & 1.024670000 & 3.170950000\end{array}$

$\begin{array}{cccc}42 & 2.624040000 & 3.059860000 & 1.209940000 \\ 42 & 0.615420000 & 4.922290000 & 1.394410000 \\ 42 & 0.609530000 & 7.086800000 & 3.686500000 \\ 42 & 2.647010000 & 4.961390000 & 3.369420000 \\ 42 & 2.694740000 & 7.195570000 & 1.385590000 \\ 42 & 0.623110000 & 0.539070000 & 5.313640000 \\ 42 & 0.626920000 & 2.825120000 & 7.522320000 \\ 42 & 2.565820000 & 0.717150000 & 7.395340000 \\ 42 & 2.790610000 & 3.021090000 & 5.454420000 \\ 42 & 0.646740000 & 5.157730000 & 5.476410000 \\ 42 & 0.611110000 & 6.975120000 & 7.467160000 \\ 42 & 2.629240000 & 4.937120000 & 7.541560000 \\ 42 & 2.613180000 & 6.917230000 & 5.651790000 \\ 7 & 8.927480000 & 7.098060000 & 5.359810000 \\ 7 & 13.099220000 & 5.026540000 & 7.437790000 \\ 7 & 13.921530000 & 7.085030000 & 5.391110000 \\ 7 & 12.899080000 & 2.948170000 & 5.410620000 \\ 7 & 13.233730000 & 0.875490000 & 7.416290000 \\ 7 & 4.919290000 & 2.908160000 & 5.403320000 \\ 7 & 10.889980000 & 2.951990000 & 3.353710000 \\ 7 & 13.950470000 & 5.017480000 & 3.395780000 \\ 7 & 6.799520000 & 7.126120000 & 3.355370000 \\ 7 & 4.821110000 & 7.110450000 & 1.373340000 \\ 7 & 13.099230000 & 7.108180000 & 1.316450000 \\ 7 & 6.867580000 & 0.870710000 & 5.379780000 \\ 7 & 13.945520000 & 2.959450000 & 1.35396000 \\ 7 & 13.382010000 & 0.849060000 & 3.381060000 \\ 7 & 0.573670000 & 3.032140000 & 1.237590000 \\ 7 & 0.434740000 & 0.886920000 & 3.294200000 \\ 7 & 0.447030000 & 7.144750000 & 5.603730000 \\ 7 & 0.449000000 & 4.911220000 & 7.495910000 \\ 7 & 0.708950000 & 2.940440000 & 5.391740000 \\ 7 & 2.658680000 & 7.113210000 & 3.477920000 \\ 7 & 0.616260000 & 7.186680000 & 1.460770000 \\ 7 & 0.448360000 & 5.024660000 & 3.430810000 \\ 7 & 0.373870000 & 0.762070000 & 7.336510000 \\ 7 & 2.684290000 & 0.847970000 & 5.302240000 \\ 7 & 10.924720000 & 2.981100000 & 7.406000000 \\ 7 & 8.935490000 & 0.883170000 & 3.336670000 \\ 7 & 10.995940000 & 7.116540000 & 3.341380000 \\ 7 & 2.658730000 & 2.993030000 & 3.270190000 \\ 1 & 14.981630000 & 5.018690000 & 3.416280000 \\ 1 & 8.905660000 & 7.106510000 & 1.254870000\end{array}$


Table S5: Coordinates and energy of $\gamma-\mathrm{Mo}_{2} \mathrm{NH}_{0.3}\left(\kappa^{1}-\mathrm{NH}\right)$, (Element Z, X, Y, Z)

$-850.228 \mathrm{eV}$

$42 \quad 4.680720000$

$42 \quad 4.870420000$

$42 \quad 6.757900000$

$42 \quad 6.857110000$

$42 \quad 8.959600000$

$42 \quad 13.018740000$

$42 \quad 8.742440000$

$42 \quad 12.994770000$

$42 \quad 11.130840000$

$42 \quad 10.990850000$

$42 \quad 4.727840000$

$42 \quad 4.741610000$

$42 \quad 6.805790000$

$42 \quad 6.861570000$

$42 \quad 8.901160000$

$42 \quad 13.038160000$

$42 \quad 8.862530000$

$42 \quad 13.012640000$

$42 \quad 10.884370000$

$42 \quad 10.978500000$

$42 \quad 4.765670000$

$42 \quad 4.767360000$

$42 \quad 6.837570000$

$42 \quad 6.966050000$

$42 \quad 8.980630000$

$42 \quad 12.973410000$

$42 \quad 8.874790000$

$42 \quad 12.993610000$

$42 \quad 11.021310000$

$42 \quad 10.738980000$

$42 \quad 4.798660000$

$42 \quad 4.719960000$

$42 \quad 6.824950000$

$42 \quad 6.775120000$

$42 \quad 8.867640000$

$42 \quad 12.988580000$

$42 \quad 8.933790000$

$42 \quad 13.017130000$

$42 \quad 10.922870000$

$42 \quad 11.011700000$

$42 \quad 0.591450000$

$42 \quad 0.629250000$

$42 \quad 2.670460000$

$\begin{array}{cc}0.892540000 & 1.279330000 \\ 2.942050000 & 3.240640000 \\ 1.000250000 & 3.201790000 \\ 2.940780000 & 1.246500000 \\ 0.853960000 & 1.271170000 \\ 0.998040000 & 1.355680000 \\ 3.021930000 & 3.276010000 \\ 2.974060000 & 3.232030000 \\ 0.882340000 & 3.309530000 \\ 2.972160000 & 1.336320000 \\ 5.014010000 & 1.303500000 \\ 6.987380000 & 3.558110000 \\ 5.004550000 & 3.325440000 \\ 7.161380000 & 1.193100000 \\ 5.031430000 & 1.297870000 \\ 4.909420000 & 1.430540000 \\ 6.938400000 & 3.149220000 \\ 7.031200000 & 3.531220000 \\ 5.034130000 & 3.272690000 \\ 7.136230000 & 1.221760000 \\ 0.723770000 & 5.471270000 \\ 2.875460000 & 7.515700000 \\ 0.837710000 & 7.488700000 \\ 3.025760000 & 5.417150000 \\ 0.943480000 & 5.486600000 \\ 0.693110000 & 5.388350000 \\ 2.922680000 & 7.522580000 \\ 2.949310000 & 7.630030000 \\ 0.861740000 & 7.445560000 \\ 3.002400000 & 5.385230000 \\ 5.019460000 & 5.499510000 \\ 7.068500000 & 7.399030000 \\ 5.004330000 & 7.560530000 \\ 7.030240000 & 5.516630000 \\ 5.030540000 & 5.443110000 \\ 5.151850000 & 5.354530000 \\ 7.104060000 & 7.452590000 \\ 7.099950000 & 7.315870000 \\ 5.086650000 & 7.460910000 \\ 7.095120000 & 5.471890000 \\ 1.126840000 & 1.255520000 \\ 2.964470000 & 3.226190000 \\ 1.024670000 & 3.170950000\end{array}$

$0.892540000 \quad 1.279330000$

$1.000250000 \quad 3.201790000$

$2.940780000 \quad 1.246500000$

$0.853960000 \quad 1.271170000$

$0.998040000 \quad 1.355680000$

3.232030000

1.336320000

1.303500000

3.558110000

3.325440000

1.193100000

1.297870000

3.149220000

3.531220000

3.272690000

1.221760000

5.471270000

7.488700000

5.417150000

5.486600000

5.388350000

7.522580000

.630030000

.4385230000

5.499510000

7.399030000

7.560530000

5.516630000

5.354530000

7.452590000

7.315870000

7.460910000

5.471890000

3.170950000

$\begin{array}{cccc}42 & 2.624040000 & 3.059860000 & 1.209940000 \\ 42 & 0.615420000 & 4.922290000 & 1.394410000 \\ 42 & 0.609530000 & 7.086800000 & 3.686500000 \\ 42 & 2.647010000 & 4.961390000 & 3.369420000 \\ 42 & 2.694740000 & 7.195570000 & 1.385590000 \\ 42 & 0.623110000 & 0.539070000 & 5.313640000 \\ 42 & 0.626920000 & 2.825120000 & 7.522320000 \\ 42 & 2.565820000 & 0.717150000 & 7.395340000 \\ 42 & 2.790610000 & 3.021090000 & 5.454420000 \\ 42 & 0.646740000 & 5.157730000 & 5.476410000 \\ 42 & 0.611110000 & 6.975120000 & 7.467160000 \\ 42 & 2.629240000 & 4.937120000 & 7.541560000 \\ 42 & 2.613180000 & 6.917230000 & 5.651790000 \\ 7 & 8.930650000 & 7.105550000 & 5.374490000 \\ 7 & 13.097720000 & 5.029200000 & 7.438260000 \\ 7 & 13.911470000 & 7.084600000 & 5.393070000 \\ 7 & 12.895100000 & 2.948260000 & 5.410530000 \\ 7 & 13.233920000 & 0.876270000 & 7.416450000 \\ 7 & 4.922480000 & 2.907600000 & 5.403830000 \\ 7 & 10.889440000 & 2.950690000 & 3.355910000 \\ 7 & 13.945140000 & 5.017930000 & 3.397620000 \\ 7 & 6.804930000 & 7.120860000 & 3.350200000 \\ 7 & 4.822050000 & 7.108670000 & 1.374440000 \\ 7 & 13.093270000 & 7.111010000 & 1.319680000 \\ 7 & 6.870100000 & 0.871870000 & 5.379720000 \\ 7 & 13.944180000 & 2.962090000 & 1.353840000 \\ 7 & 13.375780000 & 0.850340000 & 3.381190000 \\ 7 & 0.573670000 & 3.032140000 & 1.237590000 \\ 7 & 0.434740000 & 0.886920000 & 3.294200000 \\ 7 & 0.447030000 & 7.144750000 & 5.603730000 \\ 7 & 0.449000000 & 4.911220000 & 7.495910000 \\ 7 & 0.708950000 & 2.940440000 & 5.391740000 \\ 7 & 2.658680000 & 7.113210000 & 3.477920000 \\ 7 & 0.616260000 & 7.186680000 & 1.460770000 \\ 7 & 0.448360000 & 5.024660000 & 3.430810000 \\ 7 & 0.373870000 & 0.762070000 & 7.336510000 \\ 7 & 2.684290000 & 0.847970000 & 5.302240000 \\ 7 & 10.924310000 & 2.983420000 & 7.407200000 \\ 7 & 8.930780000 & 0.878270000 & 3.334550000 \\ 7 & 10.982190000 & 7.114860000 & 3.334240000 \\ 7 & 2.658730000 & 2.993030000 & 3.270190000 \\ 1 & 14.976620000 & 5.018120000 & 3.414530000\end{array}$


Table S6: Coordinates and energy of $\gamma-\mathrm{Mo}_{2} \mathrm{NH}_{0.3}\left(\mu^{6}-\mathrm{MoH}\right.$ Interstitial), (Element $\left.\mathrm{Z}, \mathrm{X}, \mathrm{Y}, \mathrm{Z}\right)$

\begin{tabular}{|c|c|c|c|c|c|c|c|}
\hline 42 & 4.689120000 & 0.899160000 & 1.279560000 & 42 & 2.624040000 & 3.059860000 & 1.209940000 \\
\hline 42 & 4.874360000 & 2.939320000 & 3.234950000 & 42 & 0.615420000 & 4.922290000 & 1.394410000 \\
\hline 42 & 6.765040000 & 1.011130000 & 3.195940000 & 42 & 0.609530000 & 7.086800000 & 3.686500000 \\
\hline 42 & 6.863300000 & 2.965820000 & 1.235950000 & 42 & 2.647010000 & 4.961390000 & 3.369420000 \\
\hline 42 & 8.950760000 & 0.867810000 & 1.266310000 & 42 & 2.694740000 & 7.195570000 & 1.385590000 \\
\hline 42 & 13.023260000 & 0.977210000 & 1.352360000 & 42 & 0.623110000 & 0.539070000 & 5.313640000 \\
\hline 42 & 8.736680000 & 3.064450000 & 3.287700000 & 42 & 0.626920000 & 2.825120000 & 7.522320000 \\
\hline 42 & 12.989440000 & 2.923660000 & 3.214370000 & 42 & 2.565820000 & 0.717150000 & 7.395340000 \\
\hline 42 & 11.149510000 & 0.884760000 & 3.313060000 & 42 & 2.790610000 & 3.021090000 & 5.454420000 \\
\hline 42 & 10.991930000 & 2.999850000 & 1.335290000 & 42 & 0.646740000 & 5.157730000 & 5.476410000 \\
\hline 42 & 4.726620000 & 5.022830000 & 1.308870000 & 42 & 0.611110000 & 6.975120000 & 7.467160000 \\
\hline 42 & 4.739890000 & 6.989040000 & 3.560010000 & 42 & 2.629240000 & 4.937120000 & 7.541560000 \\
\hline 42 & 6.806870000 & 5.001630000 & 3.315850000 & 42 & 2.613180000 & 6.917230000 & 0000 \\
\hline 42 & 6.865650000 & 7.141540000 & 1.203090000 & 7 & 8.927090000 & 7.088620000 & 5.366980000 \\
\hline 42 & 8.947270000 & 5.018890000 & 1.265820000 & 7 & 13.241420000 & 5.026610000 & 7.428830000 \\
\hline 42 & 12.999750000 & 4.881620000 & 1.352820000 & 7 & 13.931070000 & 7.103340000 & 5.402250000 \\
\hline 42 & 8.867830000 & 6.906180000 & 3.222190000 & 7 & 12.899580000 & 2.957150000 & 5.402900000 \\
\hline 42 & 13.012950000 & 7.130380000 & 3.561190000 & 7 & 13.240700000 & 0.870980000 & 7.422550000 \\
\hline 42 & 10.960550000 & 5.026730000 & 3.315690000 & 7 & 4.916440000 & 2.906630000 & 5.397200000 \\
\hline 42 & 10.979620000 & 7.104690000 & 000 & 7 & 170000 & 960000 & 20000 \\
\hline 42 & 4.765090000 & 0.721560000 & 0000 & 7 & 13.259830000 & 5.032380000 & 3.384290000 \\
\hline 42 & 4.759450000 & 2.880050000 & 0000 & 7 & 0000 & 7.130160000 & 3.355130000 \\
\hline 42 & 6.825070000 & 0.837850000 & 7.494510000 & 7 & 4.824580000 & 7.118350000 & 1.372190000 \\
\hline 42 & 6.958560000 & 3.021750000 & 5.430850000 & 7 & 13.088200000 & 7.088090000 & 1.332190000 \\
\hline 42 & 8.975800000 & 0.946480000 & 5.479830000 & 7 & 6.865970000 & 0.868510000 & 5.382900000 \\
\hline 42 & 12.977860000 & 0.721780000 & 5.400690000 & 7 & 13.949870000 & 2.927070000 & 1.349190000 \\
\hline 42 & 8.880530000 & 2.916320000 & 7.522490000 & 7 & 13.425120000 & 0.873420000 & 3.383300000 \\
\hline 42 & 13.008180000 & 0000 & 00 & 7 & 70000 & 3.0 & 90000 \\
\hline 42 & 11.006300000 & 0.843740000 & 7.450530000 & 7 & 0.434740000 & 0.886920000 & 3.294200000 \\
\hline 42 & 10.746000000 & 2.982110000 & 5.389720000 & 7 & 0.447030000 & 7.144750000 & 5.603730000 \\
\hline 42 & 4.789390000 & 5.019540000 & 5.491270000 & 7 & 0.449000000 & 4.911220000 & 7.495910000 \\
\hline 42 & 4.713920000 & 7.075490000 & 7.398740000 & 7 & 0.708950000 & 2.940440000 & 5.391740000 \\
\hline 42 & 6.794460000 & 4.993750000 & 7.553780000 & 7 & 2.658680000 & 7.113210000 & 3.477920000 \\
\hline 42 & 6.771960000 & 7.017080000 & 5.518400000 & 7 & 0.616260000 & 7.186680000 & 1.460770000 \\
\hline 42 & 8.887580000 & 5.007120000 & 5.459300000 & 7 & 0.448360000 & 5.024660000 & 3.430810000 \\
\hline 42 & 12.962520000 & 5.187930000 & 5.407660000 & 7 & 0.373870000 & 0.762070000 & 7.336510000 \\
\hline 42 & 8.942010000 & 7.094560000 & 7.419160000 & 7 & 2.684290000 & 0.847970000 & 5.302240000 \\
\hline 42 & 13.041000000 & 7.111260000 & 7.328280000 & 7 & 10.936880000 & 2.949890000 & 7.412020000 \\
\hline 42 & 11.002310000 & 5.056570000 & 7.439080000 & 7 & 8.949190000 & 0.898410000 & 3.336880000 \\
\hline 42 & 11.019740000 & 7.100600000 & 5.483460000 & 7 & 10.973710000 & 7.129770000 & 3.338330000 \\
\hline 42 & 0.591450000 & 1.126840000 & 1.255520000 & 7 & 2.658730000 & 2.993030000 & 3.270190000 \\
\hline 42 & 0.629250000 & 2.964470000 & 3.226190000 & 1 & 8.911390000 & 7.102800000 & 1.261380000 \\
\hline
\end{tabular}


Table S7: Coordinates and energy of $\gamma-\mathrm{Mo}_{2} \mathrm{NH}_{0.7}\left(\kappa^{1}-\mathrm{MoH}, \kappa^{1}-\mathrm{NH}\right)$, (Element Z, X, Y, Z) $-853.378 \mathrm{eV}$

$\begin{array}{cccc}42 & 4.677990000 & 0.893430000 & 1.276330000 \\ 42 & 4.866840000 & 2.947190000 & 3.24196000 \\ 42 & 6.742010000 & 1.005930000 & 3.196670000 \\ 42 & 6.847370000 & 2.951410000 & 1.241360000 \\ 42 & 8.943120000 & 0.868320000 & 1.262170000 \\ 42 & 12.997300000 & 0.995580000 & 1.354410000 \\ 42 & 8.717010000 & 3.030870000 & 3.278380000 \\ 42 & 12.971270000 & 2.955600000 & 3.231670000 \\ 42 & 11.108170000 & 0.901790000 & 3.306270000 \\ 42 & 10.967070000 & 2.972170000 & 1.325960000 \\ 42 & 4.723870000 & 5.015100000 & 1.297600000 \\ 42 & 4.737370000 & 6.989650000 & 3.553210000 \\ 42 & 6.799860000 & 5.013350000 & 3.328520000 \\ 42 & 6.859590000 & 7.160530000 & 1.188830000 \\ 42 & 8.896030000 & 5.032780000 & 1.290910000 \\ 42 & 13.013070000 & 4.918340000 & 1.444010000 \\ 42 & 8.851060000 & 6.959770000 & 3.148400000 \\ 42 & 12.998930000 & 6.994610000 & 3.539870000 \\ 42 & 10.837940000 & 5.049260000 & 3.242050000 \\ 42 & 10.963590000 & 7.150440000 & 1.215990000 \\ 42 & 4.762070000 & 0.727600000 & 5.468120000 \\ 42 & 4.767700000 & 2.879140000 & 7.512880000 \\ 42 & 6.831840000 & 0.844860000 & 7.485150000 \\ 42 & 6.964660000 & 3.033690000 & 5.414190000 \\ 42 & 8.975580000 & 0.949480000 & 5.485920000 \\ 42 & 12.954020000 & 0.733010000 & 5.383100000 \\ 42 & 8.876550000 & 2.932030000 & 7.510700000 \\ 42 & 12.986850000 & 2.948440000 & 7.646980000 \\ 42 & 11.025660000 & 0.866750000 & 7.435020000 \\ 42 & 10.740000000 & 3.006710000 & 5.375660000 \\ 42 & 4.794480000 & 5.021910000 & 5.497320000 \\ 42 & 4.719820000 & 7.069490000 & 7.395980000 \\ 42 & 6.830330000 & 5.009900000 & 7.550260000 \\ 42 & 6.772770000 & 7.038410000 & 5.514900000 \\ 42 & 8.869730000 & 5.048670000 & 5.430840000 \\ 42 & 13.066360000 & 5.070240000 & 5.382700000 \\ 42 & 8.931350000 & 7.113640000 & 7.450060000 \\ 42 & 13.005540000 & 7.104900000 & 7.323420000 \\ 42 & 10.926590000 & 5.101470000 & 7.441060000 \\ 42 & 11.009580000 & 7.122240000 & 5.471240000 \\ 42 & 0.591450000 & 1.126840000 & 1.255520000 \\ 42 & 0.629250000 & 2.964470000 & 3.226190000 \\ 42 & 2.670460000 & 1.024670000 & 3.170950000\end{array}$

$\begin{array}{cccc}42 & 2.624040000 & 3.059860000 & 1.209940000 \\ 42 & 0.615420000 & 4.922290000 & 1.394410000 \\ 42 & 0.609530000 & 7.086800000 & 3.686500000 \\ 42 & 2.647010000 & 4.961390000 & 3.369420000 \\ 42 & 2.694740000 & 7.195570000 & 1.385590000 \\ 42 & 0.623110000 & 0.539070000 & 5.313640000 \\ 42 & 0.626920000 & 2.825120000 & 7.522320000 \\ 42 & 2.565820000 & 0.717150000 & 7.395340000 \\ 42 & 2.790610000 & 3.021090000 & 5.454420000 \\ 42 & 0.646740000 & 5.157730000 & 5.476410000 \\ 42 & 0.611110000 & 6.975120000 & 7.467160000 \\ 42 & 2.629240000 & 4.937120000 & 7.541560000 \\ 42 & 2.613180000 & 6.917230000 & 5.651790000 \\ 7 & 8.936970000 & 7.117970000 & 5.369800000 \\ 7 & 13.070430000 & 5.040590000 & 7.479220000 \\ 7 & 13.844070000 & 7.149580000 & 5.407640000 \\ 7 & 12.886930000 & 2.925390000 & 5.408110000 \\ 7 & 13.229580000 & 0.880540000 & 7.417440000 \\ 7 & 4.921070000 & 2.910610000 & 5.402290000 \\ 7 & 10.863610000 & 2.971300000 & 3.343650000 \\ 7 & 13.945120000 & 5.032440000 & 3.376640000 \\ 7 & 6.797640000 & 7.129200000 & 3.347550000 \\ 7 & 4.821520000 & 7.109120000 & 1.371130000 \\ 7 & 13.071060000 & 7.116840000 & 1.326710000 \\ 7 & 6.866410000 & 0.878140000 & 5.378320000 \\ 7 & 13.922210000 & 2.978310000 & 1.365160000 \\ 7 & 13.347900000 & 0.824540000 & 3.375090000 \\ 7 & 0.573670000 & 3.032140000 & 1.237590000 \\ 7 & 0.434740000 & 0.886920000 & 3.294200000 \\ 7 & 0.447030000 & 7.144750000 & 5.603730000 \\ 7 & 0.449000000 & 4.911220000 & 7.495910000 \\ 7 & 0.708950000 & 2.940440000 & 5.391740000 \\ 7 & 2.658680000 & 7.113210000 & 3.477920000 \\ 7 & 0.616260000 & 7.186680000 & 1.460770000 \\ 7 & 0.448360000 & 5.024660000 & 3.430810000 \\ 7 & 0.373870000 & 0.762070000 & 7.336510000 \\ 7 & 2.684290000 & 0.847970000 & 5.302240000 \\ 7 & 10.924640000 & 2.994470000 & 7.397450000 \\ 7 & 8.912600000 & 0.892150000 & 3.326410000 \\ 7 & 10.974280000 & 7.130620000 & 3.330530000 \\ 7 & 2.658730000 & 2.993030000 & 3.270190000 \\ 1 & 14.972950000 & 5.010080000 & 3.374020000 \\ 1 & 14.729760000 & 4.769670000 & 5.724770000\end{array}$


Table S8: Coordinates and energy of $\gamma-\mathrm{Mo}_{2} \mathrm{NH}_{0.7}\left(\kappa{ }^{1}--\mathrm{NH}_{2}\right),($ Element $\mathrm{Z}, \mathrm{X}, \mathrm{Y}, \mathrm{Z})$

\begin{tabular}{|c|c|c|c|c|c|c|c|}
\hline \multicolumn{8}{|c|}{$-853.296 \mathrm{eV}$} \\
\hline \multicolumn{8}{|c|}{4.681490000} \\
\hline 42 & 4.866920000 & 2.941480000 & 3.243930000 & 42 & 0.615420000 & 4.922290000 & 1.394410000 \\
\hline 42 & 6.760800000 & 1.007790000 & 3.190490000 & 42 & 0.609530000 & 7.086800000 & 3.686500000 \\
\hline 42 & 6.862960000 & 2.953640000 & 1.247700000 & 42 & 2.647010000 & 4.961390000 & 3.369420000 \\
\hline 42 & 8.965250000 & 0.857480000 & 1.260580000 & 42 & 2.694740000 & 7.195570000 & 1.385590000 \\
\hline 42 & 13.037870000 & 1.042890000 & 1.352920000 & 42 & 0.623110000 & 0.539070000 & 5.313640000 \\
\hline 42 & 8.759610000 & 3.031150000 & 3.259020000 & 42 & 0.626920000 & 2.825120000 & 7.522320000 \\
\hline 42 & 13.045670000 & 2.999860000 & 3.265120000 & 42 & 2.565820000 & 0.717150000 & 7.395340000 \\
\hline 42 & 11.133830000 & 0.893700000 & 3.292270000 & 42 & 2.790610000 & 3.021090000 & 5.454420000 \\
\hline 42 & 10.976740000 & 2.956160000 & 1.332890000 & 42 & 0.646740000 & 5.157730000 & 5.476410000 \\
\hline 42 & 4.721300000 & 5.017290000 & 1.308050000 & 42 & 0.611110000 & 6.975120000 & 7.467160000 \\
\hline 42 & 4.734270000 & 6.989400000 & 3.559980000 & 42 & 2.629240000 & 4.937120000 & 60000 \\
\hline 42 & 6.797860000 & 5.002920000 & 3.313840000 & 42 & 2.613180000 & 6.917230000 & 90000 \\
\hline 42 & 6.857880000 & 7.160530000 & 1.178980000 & 7 & 8.932520000 & 7.100440000 & 5.367880000 \\
\hline 42 & 8.929970000 & 5.050040000 & 0000 & 7 & 110000 & 5.026840000 & 7.349320000 \\
\hline 42 & 12.956220000 & 4.979190000 & 1.373350000 & 7 & 13.770500000 & 7.079350000 & 5.341650000 \\
\hline 42 & 8.840940000 & 6.938540000 & 3.142530000 & 7 & 12.821200000 & 2.915120000 & 5.428390000 \\
\hline 42 & 12.944130000 & 000 & 000 & 7 & 13.1 & 0000 & 0000 \\
\hline 42 & 10.950490000 & 000 & 3.27 & 7 & 00 & 2.90 & 5.40 \\
\hline 42 & 10.979780000 & 0000 & 000 & 7 & 0000 & 0000 & 0000 \\
\hline 42 & 4.762870000 & 0.718 & 000 & 7 & 14.6 & 60000 & 50000 \\
\hline 42 & 4.763810000 & 2.878 & 7.50 & 7 & 0000 & 7.126050000 & 20000 \\
\hline 42 & 6.837270000 & 0.846570000 & 7.49 & 7 & 4.819660000 & 7.112610000 & 1.372790000 \\
\hline 42 & 6.965510000 & 3.034130000 & 0000 & 7 & 13.142760000 & 7.110890000 & 1.316370000 \\
\hline 42 & 8.962550000 & 0.934770000 & 50000 & 7 & & 0.864810000 & 5.376200000 \\
\hline 42 & 900000 & & 5.3 & 7 & 13. & & 0000 \\
\hline 42 & 8.874150000 & 2.93 & 7.5 & 7 & 13.4 & 0.9 & 10000 \\
\hline 42 & 12.995990000 & 2.971 & 0000 & 7 & 0000 & 40000 & 1.237590000 \\
\hline 42 & 10.992050000 & 0.845590000 & 0000 & 7 & 0.434 & 0.886920000 & 3.294200000 \\
\hline 42 & 10.689700000 & 2.998750000 & 5.391 & 7 & 0.447030000 & 7.144750000 & 5.603730000 \\
\hline 42 & 4.795680000 & 5.019500000 & 5.493140000 & 7 & 0.449000000 & 4.911220000 & 7.495910000 \\
\hline 42 & 4.713870000 & 7.069450000 & 7.395120000 & 7 & 0.708950000 & 2.940440000 & 5.391740000 \\
\hline 42 & 6.810010000 & 4.988250000 & 7.564030000 & 7 & 2.658680000 & 7.113210000 & 3.477920000 \\
\hline 42 & 6.767700000 & 7.02 & & 7 & & 7.186680000 & \\
\hline 42 & 8.852690000 & 5.023 & 5.42 & 7 & 0.448 & 5.024660000 & 10000 \\
\hline 42 & 12.966640000 & 5.072 & 000 & 7 & 0.373 & 0.762070000 & 7.336510000 \\
\hline 42 & 8.936530000 & 7.079330000 & 0000 & 7 & 2.684290000 & 0.847970000 & 5.302240000 \\
\hline 42 & 13.024720000 & 7.081650000 & 7.305300000 & 7 & 10.923980000 & 2.969210000 & 7.403570000 \\
\hline 42 & 11.015040000 & 5.077680000 & 7.450220000 & 7 & 8.951070000 & 0.882310000 & 3.328430000 \\
\hline 42 & 10.992730000 & 7.103780000 & 5.464990000 & 7 & 10.944580000 & 7.118930000 & 3.334900000 \\
\hline 42 & 0.591450000 & 1.126840000 & 1.255520000 & 7 & 2.658730000 & 2.993030000 & 3.270190000 \\
\hline 42 & 0.629250000 & 2.964470000 & 3.226190000 & 1 & 15.248270000 & 4.773900000 & 3.536450000 \\
\hline 40 & 2.670460000 & 1.024670000 & 3.170950000 & 1 & 15.261770000 & 3.614190000 & 4.726930000 \\
\hline
\end{tabular}


Table S9: Coordinates and energy of $\gamma-\mathrm{Mo}_{2} \mathrm{NH}_{0.3}$ ( $\mu^{4}$-interstitial), (Element $\left.\mathrm{Z}, \mathrm{X}, \mathrm{Y}, \mathrm{Z}\right)$

$\begin{array}{cccc}-22.552458365 & & \\ 42 & 5.629000000 & 0.994250000 & 1.085890000 \\ 42 & 5.695700000 & 3.223450000 & 3.159590000 \\ 42 & 7.737920000 & 1.134010000 & 3.041610000 \\ 42 & 7.534600000 & 3.077500000 & 1.039220000 \\ 42 & 9.619520000 & 1.025050000 & 1.013510000 \\ 42 & 13.702680000 & 1.185640000 & 1.093690000 \\ 42 & 9.597130000 & 3.050960000 & 3.025960000 \\ 42 & 13.700520000 & 3.245900000 & 2.964570000 \\ 42 & 11.616830000 & 0.957250000 & 3.012050000 \\ 42 & 11.688890000 & 3.078180000 & 1.013120000 \\ 42 & 5.559710000 & 5.085370000 & 1.227550000 \\ 42 & 5.581080000 & 6.948610000 & 3.213550000 \\ 42 & 7.733940000 & 5.088370000 & 3.056300000 \\ 42 & 7.592980000 & 7.230760000 & 1.023400000 \\ 42 & 9.634260000 & 5.129180000 & 1.034500000 \\ 42 & 13.786310000 & 5.085580000 & 1.159410000 \\ 42 & 9.744110000 & 7.271040000 & 3.062790000 \\ 42 & 13.684160000 & 7.159000000 & 3.210330000 \\ 42 & 11.563660000 & 5.224800000 & 2.992880000 \\ 42 & 11.726610000 & 7.250700000 & 1.049490000 \\ 42 & 5.638970000 & 0.649370000 & 5.153210000 \\ 42 & 5.645140000 & 3.082970000 & 7.228360000 \\ 42 & 7.574970000 & 0.992470000 & 7.258000000 \\ 42 & 7.746750000 & 3.238990000 & 5.228340000 \\ 42 & 9.798530000 & 1.149580000 & 5.273920000 \\ 42 & 13.552780000 & 0.764750000 & 5.141470000 \\ 42 & 9.654130000 & 3.079700000 & 7.403060000 \\ 42 & 13.674970000 & 3.123440000 & 7.358760000 \\ 42 & 11.742290000 & 0.989020000 & 7.260690000 \\ 42 & 11.570750000 & 3.120810000 & 5.247370000 \\ 42 & 5.601370000 & 5.239590000 & 5.104560000\end{array}$

$\begin{array}{cccc}42 & 5.580980000 & 7.213220000 & 7.066190000 \\ 42 & 7.608320000 & 5.175580000 & 7.263850000 \\ 42 & 7.626060000 & 7.118260000 & 5.240890000 \\ 42 & 9.755360000 & 5.115970000 & 5.304970000 \\ 42 & 13.678850000 & 5.381870000 & 5.090900000 \\ 42 & 9.667110000 & 7.267580000 & 7.297850000 \\ 42 & 13.721620000 & 7.271360000 & 7.013400000 \\ 42 & 11.672850000 & 5.227380000 & 7.291330000 \\ 42 & 11.704650000 & 7.255410000 & 5.184280000 \\ 7 & 9.695530000 & 7.278530000 & 5.198590000 \\ 7 & 13.799640000 & 5.190080000 & 7.205220000 \\ 7 & 14.618360000 & 7.245150000 & 5.101510000 \\ 7 & 5.298820000 & 3.057610000 & 1.135180000 \\ 7 & 5.573810000 & 1.032160000 & 3.132870000 \\ 7 & 4.620160000 & 7.230140000 & 5.074170000 \\ 7 & 5.443140000 & 5.154610000 & 7.221480000 \\ 7 & 13.952570000 & 3.117560000 & 5.165300000 \\ 7 & 13.915260000 & 1.045970000 & 7.162940000 \\ 7 & 5.661640000 & 3.087930000 & 5.188310000 \\ 7 & 11.655620000 & 3.099860000 & 3.161290000 \\ 7 & 14.709850000 & 5.174200000 & 3.165170000 \\ 7 & 7.645880000 & 7.225880000 & 3.107440000 \\ 7 & 5.441530000 & 7.254860000 & 1.087390000 \\ 7 & 4.662150000 & 5.113250000 & 3.117620000 \\ 7 & 5.346180000 & 1.021490000 & 7.151470000 \\ 7 & 13.742720000 & 7.291950000 & 1.065220000 \\ 7 & 7.697140000 & 1.047510000 & 5.164220000 \\ 7 & 14.611780000 & 3.116070000 & 1.114160000 \\ 7 & 13.773280000 & 1.014660000 & 3.125310000 \\ 1 & 14.982740000 & 3.008650000 & 5.210170000 \\ 1 & 15.738200000 & 5.177660000 & 3.191240000\end{array}$


Table S10: Coordinates and energy of $\gamma-\mathrm{Mo}_{2} \mathrm{~N}(\mathrm{OH})_{0.3,}($ Element $\mathrm{Z}, \mathrm{X}, \mathrm{Y}, \mathrm{Z})$

\begin{tabular}{|c|c|c|c|c|c|c|c|}
\hline \multicolumn{8}{|c|}{$-851.73167763 \mathrm{eV}$} \\
\hline 42 & 4.686270000 & 0.893430000 & 1.268970000 & 42 & 2.624040000 & 3.059860000 & 1.209940000 \\
\hline 42 & 4.871180000 & 2.945180000 & 3.235920000 & 42 & 0.615420000 & 4.922290000 & 1.394410000 \\
\hline 42 & 6.742830000 & 1.008400000 & 3.188980000 & 42 & 0.609530000 & 7.086800000 & 3.686500000 \\
\hline 42 & 6.849560000 & 2.954660000 & 1.215530000 & 42 & 2.647010000 & 4.961390000 & 3.369420000 \\
\hline 42 & 8.947610000 & 0.853360000 & 1.262850000 & 42 & 2.694740000 & 7.195570000 & 1.385590000 \\
\hline 42 & 13.006500000 & 0.932050000 & 1.359250000 & 42 & 0.623110000 & 0.539070000 & 5.313640000 \\
\hline 42 & 8.691560000 & 3.040980000 & 3.286750000 & 42 & 0.626920000 & 2.825120000 & 7.522320000 \\
\hline 42 & 12.874110000 & 2.813540000 & 3.280770000 & 42 & 2.565820000 & 0.717150000 & 7.395340000 \\
\hline 42 & 11.112680000 & 0.870890000 & 3.301820000 & 42 & 2.790610000 & 3.021090000 & 5.454420000 \\
\hline 42 & 10.926400000 & 2.973930000 & 1.312190000 & 42 & 0.646740000 & 5.157730000 & 5.476410000 \\
\hline 42 & 4.729730000 & 5.013930000 & 1.299060000 & 42 & 0.611110000 & 6.975120000 & 7.467160000 \\
\hline 42 & 4.738110000 & 6.984870000 & 3.556940000 & 42 & 2.629240000 & 4.937120000 & 7.541560000 \\
\hline 42 & 6.803460000 & 5.007290000 & 3.323670000 & 42 & 2.613180000 & 6.917230000 & 5.651790000 \\
\hline 42 & 6.858020000 & 7.134360000 & 1.208520000 & 7 & 8.938670000 & 7.081570000 & 5.355990000 \\
\hline 42 & 8.902850000 & 5.003710000 & 1.268270000 & 7 & 13.115010000 & 80000 & 90000 \\
\hline 42 & 12.985970000 & 4.885950000 & 1.459 & 7 & 13.939950000 & 7.085100000 & 10000 \\
\hline 42 & 8.868230000 & 6.929140000 & 3.217690000 & 7 & 12.935460000 & 2.947480000 & 5.401760000 \\
\hline 42 & 13.034430000 & 7.028420000 & 3.559210000 & 7 & 13.238380000 & 0.857190000 & 7.416120000 \\
\hline 42 & 10.934370000 & 5.024340000 & 3.271470000 & 7 & 4.910310000 & 2.907310000 & 5.400080000 \\
\hline 42 & 10.981920000 & 7.093140000 & 1.235280000 & 7 & 10.807980000 & 2.947850000 & 3.333930000 \\
\hline 42 & 4.764050000 & 0.725240000 & 5.476340000 & 7 & 6.805550000 & 7.133430000 & 3.353830000 \\
\hline 42 & 4.757730000 & 2.881710000 & 7.515020000 & 7 & 4.820090000 & 7.111370000 & 1.371590000 \\
\hline 42 & 6.841620000 & 0.819160000 & 7.487780000 & 7 & 13.093080000 & 7.059370000 & 1.331220000 \\
\hline 42 & 6.948380000 & 3.011450000 & 5.427710000 & 7 & 6.867000000 & 0.867430000 & 5.374400000 \\
\hline 42 & 8.976310000 & 0.94 & & 7 & 13.8 & & 0000 \\
\hline 42 & 12.988880000 & 0.690440000 & 5.38 & 7 & 13.42 & 0.750360000 & 170000 \\
\hline 42 & 8.878960000 & 2.906900000 & 7.500 & 7 & 0.573670000 & 3.032140000 & 1.237590000 \\
\hline 42 & 12.989680000 & 2.924660000 & 7.666730000 & 7 & 0.434740000 & 0.886920000 & 3.294200000 \\
\hline 42 & 11.016060000 & 0.830010000 & 7.434080000 & 7 & 0.447030000 & 7.144750000 & 5.603730000 \\
\hline 42 & 10.757790000 & 3.004490000 & 5.372950000 & 7 & 0.449000000 & 4.911220000 & 7.495910000 \\
\hline 42 & 4.793020000 & 5.020670000 & 5.496830000 & 7 & 0.708950000 & 2.940440000 & 5.391740000 \\
\hline 42 & 4.715510000 & 7.072280000 & 7.394670000 & 7 & 2.658680000 & 7.113210000 & 3.477920000 \\
\hline 42 & 6.813810000 & 5.008330000 & 7.535660000 & 7 & 0.616260000 & 7.186680000 & 1.460770000 \\
\hline 42 & 6.772880000 & 7.028590000 & 5.510230000 & 7 & & 5.024660000 & 3.430810000 \\
\hline 42 & 8.865000000 & 5.00 & & 7 & & 0.762 & 7.336510000 \\
\hline 42 & 13.021150000 & 5.140 & 5.36 & 7 & & 0.847 & 5.302240000 \\
\hline 42 & 8.945060000 & 7.095130000 & 7.404180000 & 7 & 10.938040000 & 2.952250000 & 7.385730000 \\
\hline 42 & 13.017840000 & 7.067730000 & 7.318130000 & 7 & 8.928370000 & 0.881570000 & 3.335670000 \\
\hline 42 & 10.955790000 & 5.063320000 & 7.439210000 & 7 & 11.008280000 & 7.108070000 & 3.338070000 \\
\hline 42 & 11.031100000 & 7.076770000 & 5.471190000 & 7 & 2.658730000 & 2.993030000 & 3.270190000 \\
\hline 42 & 0.591450000 & 1.126840000 & 1.255520000 & 1 & 15.232180000 & 5.457860000 & 3.467660000 \\
\hline 42 & 0.629250000 & 2.964470000 & 3.226190000 & 1 & 8.907190000 & 7.085450000 & 1.252990000 \\
\hline 42 & 2.670460000 & 1.024670000 & 3.170950000 & 8 & 14.275330000 & 5.254350000 & 3.393760000 \\
\hline
\end{tabular}


Table S11: Coordinates and energy of $\kappa^{1}$-Bound Crotonaldehyde to $\gamma-\mathrm{Mo}_{2} \mathrm{~N}+2 \mathrm{H}$, (Element $\mathrm{Z}, \mathrm{X}$, $\mathrm{Y}, \mathrm{Z})$

$\begin{array}{cccc}-918.399 \mathrm{eV} & & \\ 42 & 4.677790000 & 0.889680000 & 1.283530000 \\ 42 & 4.852250000 & 2.966940000 & 3.278240000 \\ 42 & 6.712260000 & 0.996100000 & 3.203600000 \\ 42 & 6.845300000 & 2.961480000 & 1.312690000 \\ 42 & 8.913150000 & 0.866040000 & 1.25299000 \\ 42 & 13.025940000 & 1.049870000 & 1.382700000 \\ 42 & 8.840680000 & 2.944020000 & 3.316310000 \\ 42 & 13.231320000 & 3.062780000 & 3.308630000 \\ 42 & 10.925380000 & 0.930230000 & 3.294480000 \\ 42 & 11.044910000 & 2.987510000 & 1.390620000 \\ 42 & 4.719310000 & 5.012220000 & 1.315140000 \\ 42 & 4.743170000 & 6.981550000 & 3.569440000 \\ 42 & 6.835420000 & 4.993790000 & 3.33899000 \\ 42 & 6.869510000 & 7.126690000 & 1.204590000 \\ 42 & 8.926100000 & 5.011040000 & 1.271200000 \\ 42 & 13.060730000 & 4.949020000 & 1.447040000 \\ 42 & 8.880780000 & 6.972250000 & 3.200430000 \\ 42 & 13.019250000 & 7.014930000 & 3.548710000 \\ 42 & 11.032930000 & 5.051950000 & 3.301050000 \\ 42 & 10.995200000 & 7.142690000 & 1.254650000 \\ 42 & 4.762860000 & 0.726810000 & 5.481070000 \\ 42 & 4.752980000 & 2.890170000 & 7.509410000 \\ 42 & 6.791450000 & 0.869390000 & 7.529260000 \\ 42 & 6.967670000 & 3.032790000 & 5.438930000 \\ 42 & 8.958800000 & 0.948080000 & 5.548310000 \\ 42 & 12.913170000 & 0.711310000 & 5.433400000 \\ 42 & 8.882920000 & 2.935510000 & 7.581890000 \\ 42 & 13.004580000 & 2.981930000 & 7.640690000 \\ 42 & 11.049900000 & 0.875960000 & 7.506980000 \\ 42 & 10.710400000 & 3.009210000 & 5.433380000 \\ 42 & 4.790010000 & 5.030180000 & 5.496020000 \\ 42 & 4.713970000 & 7.062820000 & 7.410400000 \\ 42 & 6.797230000 & 4.985160000 & 7.564110000 \\ 42 & 6.770720000 & 7.033210000 & 5.544110000 \\ 42 & 8.857080000 & 5.025760000 & 5.518700000 \\ 42 & 12.977510000 & 5.169500000 & 5.384120000 \\ 42 & 8.933830000 & 7.128380000 & 7.508260000 \\ 42 & 13.033350000 & 7.124180000 & 7.341570000 \\ 42 & 10.956740000 & 5.095670000 & 7.512680000 \\ 42 & 11.016080000 & 7.117180000 & 5.511990000 \\ 42 & 0.591450000 & 1.126840000 & 1.255520000 \\ 42 & 0.629250000 & 2.964470000 & 3.226190000 \\ 42 & 2.670460000 & 1.024670000 & 3.170950000 \\ 42 & 2.624040000 & 3.059860000 & 1.209940000 \\ 42 & 0.615420000 & 4.922290000 & 1.394410000 \\ 42 & 0.609530000 & 7.086800000 & 3.686500000 \\ 42 & 2.647010000 & 4.961390000 & 3.369420000 \\ 42 & 2.694740000 & 7.195570000 & 1.385590000 \\ 42 & 0.623110000 & 0.539070000 & 5.313640000\end{array}$

$\begin{array}{cccc}42 & 0.626920000 & 2.825120000 & 7.522320000 \\ 42 & 2.565820000 & 0.717150000 & 7.395340000 \\ 42 & 2.790610000 & 3.021090000 & 5.454420000 \\ 42 & 0.646740000 & 5.157730000 & 5.476410000 \\ 42 & 0.611110000 & 6.975120000 & 7.467160000 \\ 42 & 2.629240000 & 4.937120000 & 7.541560000 \\ 42 & 2.613180000 & 6.917230000 & 5.651790000 \\ 7 & 8.932670000 & 7.108940000 & 5.419690000 \\ 7 & 13.142710000 & 5.056880000 & 7.468490000 \\ 7 & 13.907620000 & 7.140330000 & 5.403640000 \\ 7 & 12.859250000 & 2.960130000 & 5.447600000 \\ 7 & 13.268320000 & 0.897460000 & 7.444090000 \\ 7 & 4.924170000 & 2.915350000 & 5.419520000 \\ 7 & 11.022110000 & 2.997240000 & 3.419110000 \\ 7 & 13.991300000 & 5.100170000 & 3.432680000 \\ 7 & 6.803940000 & 7.102450000 & 3.366800000 \\ 7 & 4.823010000 & 7.105400000 & 1.381650000 \\ 7 & 13.109580000 & 7.151250000 & 1.352830000 \\ 7 & 6.860060000 & 0.875970000 & 5.416320000 \\ 7 & 14.005570000 & 2.980640000 & 1.291190000 \\ 7 & 13.044410000 & 0.873900000 & 3.406980000 \\ 7 & 0.573670000 & 3.032140000 & 1.237590000 \\ 7 & 0.434740000 & 0.886920000 & 3.294200000 \\ 7 & 0.447030000 & 7.144750000 & 5.603730000 \\ 7 & 0.449000000 & 4.911220000 & 7.495910000 \\ 7 & 0.708950000 & 2.940440000 & 5.391740000 \\ 7 & 2.658680000 & 7.113210000 & 3.477920000 \\ 7 & 0.616260000 & 7.186680000 & 1.460770000 \\ 7 & 0.448360000 & 5.024660000 & 3.430810000 \\ 7 & 0.373870000 & 0.762070000 & 7.336510000 \\ 7 & 2.684290000 & 0.847970000 & 5.302240000 \\ 7 & 10.928640000 & 2.989140000 & 7.454630000 \\ 7 & 8.797100000 & 0.838430000 & 3.365920000 \\ 7 & 10.987710000 & 7.143690000 & 3.357910000 \\ 7 & 2.658730000 & 2.993030000 & 3.270190000 \\ 1 & 15.019940000 & 5.155870000 & 3.510600000 \\ 1 & 16.229890000 & 3.332340000 & 2.170600000 \\ 1 & 17.637250000 & 2.241310000 & 4.758480000 \\ 1 & 18.661950000 & 3.376040000 & 2.067610000 \\ 1 & 20.116250000 & 2.358830000 & 4.631160000 \\ 1 & 20.678950000 & 2.014300000 & 2.972530000 \\ 1 & 20.711280000 & 3.669590000 & 3.570970000 \\ 1 & 8.838310000 & 4.978370000 & 3.369210000 \\ 6 & 18.750090000 & 2.952610000 & 3.075630000 \\ 6 & 17.603020000 & 2.669530000 & 3.750860000 \\ 6 & 20.124370000 & 2.735170000 & 3.598830000 \\ 6 & 16.314280000 & 2.918600000 & 3.192760000 \\ 8 & 15.241210000 & 2.659460000 & 3.832780000\end{array}$


Table S12: Coordinates and energy of "Alkoxide-bound to $\gamma-\mathrm{Mo}_{2} \mathrm{~N}$ ", (Element Z, X, Y, Z)

$\begin{array}{cccc}-918.685 \mathrm{eV} & & \\ 42 & 4.675230000 & 0.889390000 & 1.279900000 \\ 42 & 4.863540000 & 2.945200000 & 3.255310000 \\ 42 & 6.736470000 & 0.992510000 & 3.209800000 \\ 42 & 6.844410000 & 2.950880000 & 1.279720000 \\ 42 & 8.935250000 & 0.843720000 & 1.261260000 \\ 42 & 13.027370000 & 1.049140000 & 1.347830000 \\ 42 & 8.788910000 & 2.989930000 & 3.263100000 \\ 42 & 13.256400000 & 3.00969000 & 3.293800000 \\ 42 & 11.013820000 & 0.970070000 & 3.288510000 \\ 42 & 11.028560000 & 2.98555000 & 1.357790000 \\ 42 & 4.720990000 & 5.016480000 & 1.309680000 \\ 42 & 4.740450000 & 6.988420000 & 3.560220000 \\ 42 & 6.812860000 & 5.002190000 & 3.325890000 \\ 42 & 6.856470000 & 7.149790000 & 1.198670000 \\ 42 & 8.914040000 & 5.042720000 & 1.311630000 \\ 42 & 13.065640000 & 4.922520000 & 1.429980000 \\ 42 & 8.868470000 & 6.910170000 & 3.161920000 \\ 42 & 13.005120000 & 7.009800000 & 3.516690000 \\ 42 & 11.016270000 & 5.050010000 & 3.271730000 \\ 42 & 10.993870000 & 7.161990000 & 1.234260000 \\ 42 & 4.761010000 & 0.724020000 & 5.470380000 \\ 42 & 4.762510000 & 2.877630000 & 7.506270000 \\ 42 & 6.812720000 & 0.854510000 & 7.502650000 \\ 42 & 6.976790000 & 3.044750000 & 5.415170000 \\ 42 & 8.961370000 & 0.941970000 & 5.508980000 \\ 42 & 12.912410000 & 0.709270000 & 5.404940000 \\ 42 & 8.859520000 & 2.942200000 & 7.576930000 \\ 42 & 12.979890000 & 2.987250000 & 7.598130000 \\ 42 & 11.023840000 & 0.879800000 & 7.474020000 \\ 42 & 10.668540000 & 3.022230000 & 5.407100000 \\ 42 & 4.798120000 & 5.023400000 & 5.492700000 \\ 42 & 4.712340000 & 7.067960000 & 7.403030000 \\ 42 & 6.797370000 & 5.000670000 & 7.567940000 \\ 42 & 6.764920000 & 7.035970000 & 5.526940000 \\ 42 & 8.850510000 & 5.028880000 & 5.467620000 \\ 42 & 12.954960000 & 5.174190000 & 5.351250000 \\ 42 & 8.921210000 & 7.113250000 & 7.470980000 \\ 42 & 13.013240000 & 7.125350000 & 7.314190000 \\ 42 & 10.921900000 & 5.111060000 & 7.493120000 \\ 42 & 10.998660000 & 7.122830000 & 5.472960000 \\ 42 & 0.591450000 & 1.126840000 & 1.255520000 \\ 42 & 0.629250000 & 2.964470000 & 3.226190000 \\ 42 & 2.670460000 & 1.024670000 & 3.170950000 \\ 42 & 2.624040000 & 3.059860000 & 1.209940000 \\ 42 & 0.615420000 & 4.922290000 & 1.394410000 \\ 42 & 0.609530000 & 7.086800000 & 3.686500000 \\ 42 & 2.647010000 & 4.961390000 & 3.369420000 \\ 42 & 2.694740000 & 7.195570000 & 1.385590000 \\ 42 & 0.623110000 & 0.539070000 & 5.313640000\end{array}$

$\begin{array}{cccc}42 & 0.626920000 & 2.825120000 & 7.522320000 \\ 42 & 2.565820000 & 0.717150000 & 7.395340000 \\ 42 & 2.790610000 & 3.021090000 & 5.454420000 \\ 42 & 0.646740000 & 5.157730000 & 5.476410000 \\ 42 & 0.611110000 & 6.975120000 & 7.467160000 \\ 42 & 2.629240000 & 4.937120000 & 7.541560000 \\ 42 & 2.613180000 & 6.917230000 & 5.651790000 \\ 7 & 8.922090000 & 7.108890000 & 5.390360000 \\ 7 & 13.093980000 & 5.063450000 & 7.444510000 \\ 7 & 13.885460000 & 7.137250000 & 5.385580000 \\ 7 & 12.817670000 & 2.969900000 & 5.410130000 \\ 7 & 13.232940000 & 0.904330000 & 7.413080000 \\ 7 & 4.928910000 & 2.908820000 & 5.405820000 \\ 7 & 10.949320000 & 3.023980000 & 3.389860000 \\ 7 & 13.962580000 & 5.113350000 & 3.405700000 \\ 7 & 6.807640000 & 7.114520000 & 3.358200000 \\ 7 & 4.817440000 & 7.108760000 & 1.376990000 \\ 7 & 13.103420000 & 7.147970000 & 1.319730000 \\ 7 & 6.860410000 & 0.880210000 & 5.395050000 \\ 7 & 13.979710000 & 2.987520000 & 1.220930000 \\ 7 & 13.133050000 & 0.855260000 & 3.372780000 \\ 7 & 0.573670000 & 3.032140000 & 1.237590000 \\ 7 & 0.434740000 & 0.886920000 & 3.294200000 \\ 7 & 0.447030000 & 7.144750000 & 5.603730000 \\ 7 & 0.449000000 & 4.911220000 & 7.495910000 \\ 7 & 0.708950000 & 2.940440000 & 5.391740000 \\ 7 & 2.658680000 & 7.113210000 & 3.477920000 \\ 7 & 0.616260000 & 7.186680000 & 1.460770000 \\ 7 & 0.448360000 & 5.024660000 & 3.430810000 \\ 7 & 0.373870000 & 0.762070000 & 7.336510000 \\ 7 & 2.684290000 & 0.847970000 & 5.302240000 \\ 7 & 10.901910000 & 3.002420000 & 7.427700000 \\ 7 & 8.858560000 & 0.852190000 & 3.347840000 \\ 7 & 10.970080000 & 7.166300000 & 3.329840000 \\ 7 & 2.658730000 & 2.993030000 & 3.270190000 \\ 1 & 14.991640000 & 5.068080000 & 3.495030000 \\ 1 & 18.319950000 & 2.304190000 & 1.657370000 \\ 1 & 16.029730000 & 1.852490000 & 2.105600000 \\ 1 & 17.691160000 & 2.426840000 & 4.667090000 \\ 1 & 15.887970000 & 0.884110000 & 3.603090000 \\ 1 & 20.035150000 & 2.940930000 & 4.182600000 \\ 1 & 20.622850000 & 1.963270000 & 2.811740000 \\ 1 & 20.283740000 & 3.679210000 & 2.575550000 \\ 6 & 18.522540000 & 2.441130000 & 2.727840000 \\ 6 & 17.514240000 & 2.283740000 & 3.594370000 \\ 6 & 19.932910000 & 2.774700000 & 3.100100000 \\ 6 & 16.119200000 & 1.898210000 & 3.208940000 \\ 8 & 15.189670000 & 2.834990000 & 3.740050000\end{array}$


Table S13: Coordinates and energy of " $\kappa^{1}-\mathrm{O}-$ crotyl alcohol-bound to $\gamma-\mathrm{Mo}_{2} \mathrm{~N}$ ", (Element Z, X, $\mathrm{Y}, \mathrm{Z})$

$-918.576 \mathrm{eV}$

$42 \quad 4.681350000$

$42 \quad 4.867680000$

$42 \quad 6.770630000$

$42 \quad 6.863250000$

$42 \quad 8.951630000$

$42 \quad 13.008730000$

$42 \quad 8.752120000$

$42 \quad 13.059740000$

$42 \quad 11.151470000$

$42 \quad 10.989850000$

$42 \quad 4.720220000$

$42 \quad 4.738310000$

$42 \quad 6.786180000$

$42 \quad 6.863980000$

$42 \quad 8.932050000$

$42 \quad 12.993390000$

$42 \quad 8.850390000$

$42 \quad 12.974720000$

$42 \quad 10.932690000$

$42 \quad 10.956540000$

$42 \quad 4.763600000$

$42 \quad 4.760070000$

$42 \quad 6.819420000$

$42 \quad 6.964800000$

$42 \quad 8.972030000$

$42 \quad 12.940620000$

$42 \quad 8.865900000$

$42 \quad 12.988400000$

$42 \quad 10.978700000$

$42 \quad 10.716140000$

$42 \quad 4.790110000$

$42 \quad 4.711110000$

$42 \quad 6.780420000$

$42 \quad 6.767260000$

$42 \quad 8.877940000$

$42 \quad 12.925500000$

$42 \quad 8.926670000$

$42 \quad 13.018420000$

$42 \quad 10.992920000$

$42 \quad 10.987930000$

$42 \quad 0.591450000$

$42 \quad 0.629250000$

$42 \quad 2.670460000$

$42 \quad 2.624040000$

$42 \quad 0.615420000$

$42 \quad 0.609530000$

$42 \quad 2.647010000$

$42 \quad 2.694740000$

$42 \quad 0.623110000$

\begin{tabular}{cc}
0.895470000 & 1.287240000 \\
2.936140000 & 3.242900000 \\
0.996860000 & 3.203370000 \\
2.948210000 & 1.258390000 \\
0.856180000 & 1.269820000 \\
0.983550000 & 1.353200000 \\
3.040700000 & 3.283760000 \\
2.915790000 & 3.234920000 \\
0.891680000 & 3.309390000 \\
3.000020000 & 1.340360000 \\
5.022370000 & 1.315570000 \\
6.988820000 & 3.566160000 \\
4.992920000 & 3.311310000 \\
7.153120000 & 1.186790000 \\
5.034170000 & 1.278500000 \\
4.877210000 & 1.352300000 \\
6.899180000 & 3.152220000 \\
7.129340000 & 3.559850000 \\
5.030160000 & 3.315060000 \\
7.118380000 & 1.224240000 \\
0.713500000 & 5.468350000 \\
2.867230000 & 7.507140000 \\
0.849400000 & 7.496590000 \\
3.023990000 & 5.421880000 \\
0.932420000 & 5.481570000 \\
0.713140000 & 5.407220000 \\
2.928920000 & 7.530590000 \\
2.946960000 & 7.613120000 \\
0.852060000 & 7.461220000 \\
2.986370000 & 5.390460000 \\
5.014840000 & 5.493970000 \\
7.074320000 & 7.405090000 \\
4.976490000 & 7.579910000 \\
7.010540000 & 5.527110000 \\
5.017530000 & 5.455460000 \\
5.195960000 & 5.414560000 \\
7.082370000 & 7.472650000 \\
7.114020000 & 7.338680000 \\
5.054530000 & 7.447430000 \\
7.095630000 & 5.490030000 \\
2.964470000 & 3.226190000 \\
1.024670000 & 3.170950000 \\
\hline .059860000 & 1.209940000 \\
\hline .968290000 & 1.394410000 \\
\hline
\end{tabular}

$\begin{array}{cccc}42 & 0.626920000 & 2.825120000 & 7.522320000 \\ 42 & 2.565820000 & 0.717150000 & 7.395340000 \\ 42 & 2.790610000 & 3.021090000 & 5.454420000 \\ 42 & 0.646740000 & 5.157730000 & 5.476410000 \\ 42 & 0.611110000 & 6.975120000 & 7.467160000 \\ 42 & 2.629240000 & 4.937120000 & 7.541560000 \\ 42 & 2.613180000 & 6.917230000 & 5.651790000 \\ 7 & 8.911440000 & 7.093310000 & 5.382150000 \\ 7 & 13.233560000 & 5.032020000 & 7.429210000 \\ 7 & 13.902070000 & 7.102300000 & 5.403070000 \\ 7 & 12.876650000 & 2.957630000 & 5.404680000 \\ 7 & 13.220930000 & 0.872870000 & 7.423540000 \\ 7 & 4.920400000 & 2.899060000 & 5.400830000 \\ 7 & 10.905730000 & 2.949560000 & 3.361150000 \\ 7 & 13.171190000 & 5.037120000 & 3.386930000 \\ 7 & 6.798460000 & 7.118730000 & 3.351710000 \\ 7 & 4.821670000 & 7.114530000 & 1.378800000 \\ 7 & 13.070000000 & 7.090870000 & 1.335990000 \\ 7 & 6.863140000 & 0.862180000 & 5.386600000 \\ 7 & 13.962500000 & 2.936440000 & 1.294490000 \\ 7 & 13.415230000 & 0.848970000 & 3.385270000 \\ 7 & 0.573670000 & 3.032140000 & 1.237590000 \\ 7 & 0.434740000 & 0.886920000 & 3.294200000 \\ 7 & 0.447030000 & 7.144750000 & 5.603730000 \\ 7 & 0.449000000 & 4.911220000 & 7.495910000 \\ 7 & 0.708950000 & 2.940440000 & 5.391740000 \\ 7 & 2.658680000 & 7.113210000 & 3.477920000 \\ 7 & 0.616260000 & 7.186680000 & 1.460770000 \\ 7 & 0.448360000 & 5.024660000 & 3.430810000 \\ 7 & 0.373870000 & 0.762070000 & 7.336510000 \\ 7 & 2.684290000 & 0.847970000 & 5.302240000 \\ 7 & 10.918180000 & 2.953310000 & 7.409910000 \\ 7 & 8.950430000 & 0.891440000 & 3.334040000 \\ 7 & 10.933640000 & 7.139750000 & 3.331710000 \\ 7 & 2.658730000 & 2.993030000 & 3.270190000 \\ 1 & 15.301160000 & 2.667720000 & 4.770230000 \\ 1 & 16.110950000 & 1.387430000 & 2.869040000 \\ 1 & 16.488860000 & 2.992030000 & 2.185320000 \\ 1 & 17.739390000 & 1.804650000 & 4.785660000 \\ 1 & 18.729390000 & 3.738610000 & 2.611090000 \\ 1 & 20.054850000 & 2.500330000 & 5.149000000 \\ 1 & 20.898580000 & 2.720650000 & 3.592560000 \\ 6 & 20.398810000 & 4.136430000 & 4.519690000 \\ & 18.785890000 & 3.123440000 & 3.518390000 \\ & 17.697910000 & 2.432830000 & 3.885310000 \\ 7 & 15.421510000 & 2.417600000 & 3.116820000 \\ 7 & & & \\ 7 & & & \\ 7 & & \end{array}$


Table S14: Coordinates and energy of "Butanal-bound to $\gamma-\mathrm{Mo}_{2} \mathrm{~N}$ ", (Element Z, X, Y, Z)

$\begin{array}{cccc}-919.103 \mathrm{eV} & & \\ 42 & 4.680060000 & 0.895940000 & 1.288690000 \\ 42 & 4.866540000 & 2.937130000 & 3.247030000 \\ 42 & 6.770740000 & 0.999430000 & 3.204770000 \\ 42 & 6.860170000 & 2.949560000 & 1.262970000 \\ 42 & 8.951010000 & 0.855740000 & 1.270990000 \\ 42 & 13.006620000 & 0.988420000 & 1.355400000 \\ 42 & 8.751700000 & 3.041200000 & 3.284120000 \\ 42 & 13.079160000 & 2.915450000 & 3.253630000 \\ 42 & 11.156830000 & 0.896790000 & 3.309100000 \\ 42 & 10.999830000 & 3.006940000 & 1.346780000 \\ 42 & 4.718200000 & 5.023580000 & 1.316930000 \\ 42 & 4.737900000 & 6.991600000 & 3.566740000 \\ 42 & 6.784180000 & 4.996330000 & 3.312490000 \\ 42 & 6.863010000 & 7.155600000 & 1.188540000 \\ 42 & 8.932300000 & 5.038320000 & 1.285940000 \\ 42 & 12.995850000 & 4.879950000 & 1.355680000 \\ 42 & 8.850540000 & 6.904880000 & 3.150510000 \\ 42 & 12.977460000 & 7.129800000 & 3.564670000 \\ 42 & 10.937520000 & 5.036160000 & 3.316510000 \\ 42 & 10.956080000 & 7.121970000 & 1.226330000 \\ 42 & 4.762210000 & 0.714200000 & 5.469470000 \\ 42 & 4.760410000 & 2.868370000 & 7.508900000 \\ 42 & 6.819650000 & 0.851220000 & 7.498610000 \\ 42 & 6.967380000 & 3.027820000 & 5.424170000 \\ 42 & 8.968860000 & 0.934450000 & 5.482030000 \\ 42 & 12.942160000 & 0.720430000 & 5.406780000 \\ 42 & 8.862330000 & 2.934360000 & 7.541530000 \\ 42 & 12.983800000 & 2.951980000 & 7.612220000 \\ 42 & 10.974090000 & 0.855480000 & 7.464120000 \\ 42 & 10.697140000 & 2.999780000 & 5.394620000 \\ 42 & 4.790810000 & 5.015660000 & 5.495000000 \\ 42 & 4.710070000 & 7.074990000 & 7.405690000 \\ 42 & 6.779410000 & 4.980380000 & 7.584210000 \\ 42 & 6.764440000 & 7.014050000 & 5.527760000 \\ 42 & 8.871150000 & 5.024580000 & 5.455640000 \\ 42 & 12.918920000 & 5.196970000 & 5.419310000 \\ 42 & 8.924490000 & 7.084710000 & 7.471610000 \\ 42 & 13.016760000 & 7.119250000 & 7.339620000 \\ 42 & 10.988620000 & 5.060870000 & 7.452930000 \\ 42 & 10.989390000 & 7.101930000 & 5.491250000 \\ 42 & 0.591450000 & 1.126840000 & 1.255520000 \\ 42 & 0.629250000 & 2.964470000 & 3.226190000 \\ 42 & 2.670460000 & 1.024670000 & 3.170950000 \\ 42 & 2.624040000 & 3.059860000 & 1.209940000 \\ 42 & 0.615420000 & 4.922290000 & 1.394410000 \\ 42 & 0.609530000 & 7.086800000 & 3.686500000 \\ 42 & 2.647010000 & 4.961390000 & 3.369420000 \\ 42 & 2.694740000 & 7.195570000 & 1.385590000 \\ 42 & 0.623110000 & 0.539070000 & 5.313640000\end{array}$

$\begin{array}{cccc}42 & 0.626920000 & 2.825120000 & 7.522320000 \\ 42 & 2.565820000 & 0.717150000 & 7.395340000 \\ 42 & 2.790610000 & 3.021090000 & 5.454420000 \\ 42 & 0.646740000 & 5.157730000 & 5.476410000 \\ 42 & 0.611110000 & 6.975120000 & 7.467160000 \\ 42 & 2.629240000 & 4.937120000 & 7.541560000 \\ 42 & 2.613180000 & 6.917230000 & 5.651790000 \\ 7 & 8.910690000 & 7.099080000 & 5.381950000 \\ 7 & 13.228390000 & 5.038360000 & 7.432060000 \\ 7 & 13.905110000 & 7.109070000 & 5.404770000 \\ 7 & 12.841900000 & 2.965220000 & 5.412820000 \\ 7 & 13.212510000 & 0.880030000 & 7.421580000 \\ 7 & 4.921730000 & 2.899400000 & 5.403880000 \\ 7 & 10.904690000 & 2.953320000 & 3.366460000 \\ 7 & 13.176330000 & 5.038770000 & 3.390780000 \\ 7 & 6.798200000 & 7.120850000 & 3.352510000 \\ 7 & 4.821080000 & 7.115480000 & 1.379440000 \\ 7 & 13.066770000 & 7.095510000 & 1.336440000 \\ 7 & 6.861750000 & 0.864990000 & 5.388030000 \\ 7 & 13.970020000 & 2.941710000 & 1.285270000 \\ 7 & 13.421020000 & 0.846340000 & 3.379490000 \\ 7 & 0.573670000 & 3.032140000 & 1.237590000 \\ 7 & 0.434740000 & 0.886920000 & 3.294200000 \\ 7 & 0.447030000 & 7.144750000 & 5.603730000 \\ 7 & 0.449000000 & 4.911220000 & 7.495910000 \\ 7 & 0.708950000 & 2.940440000 & 5.391740000 \\ 7 & 2.658680000 & 7.113210000 & 3.477920000 \\ 7 & 0.616260000 & 7.186680000 & 1.460770000 \\ 7 & 0.448360000 & 5.024660000 & 3.430810000 \\ 7 & 0.373870000 & 0.762070000 & 7.336510000 \\ 7 & 2.684290000 & 0.847970000 & 5.302240000 \\ 7 & 10.914130000 & 2.958120000 & 7.414410000 \\ 7 & 8.951610000 & 0.894120000 & 3.335470000 \\ 7 & 10.934730000 & 7.146170000 & 3.333860000 \\ 7 & 2.658730000 & 2.993030000 & 3.270190000 \\ 1 & 17.635990000 & 3.447030000 & 4.561930000 \\ 1 & 18.288570000 & 4.268390000 & 2.229910000 \\ 1 & 16.058410000 & 2.187340000 & 2.184670000 \\ 1 & 17.940330000 & 1.785320000 & 4.008770000 \\ 1 & 18.574010000 & 2.599930000 & 1.732340000 \\ 1 & 20.401760000 & 2.393880000 & 3.484660000 \\ 1 & 20.737280000 & 3.714370000 & 2.342940000 \\ 1 & 20.115240000 & 4.080030000 & 3.966310000 \\ 6 & 18.614800000 & 3.282050000 & 2.598040000 \\ 6 & 17.629630000 & 2.795810000 & 3.674400000 \\ 6 & 20.045110000 & 3.372060000 & 3.125810000 \\ 6 & 16.232360000 & 2.663890000 & 3.171700000 \\ 8 & 15.256570000 & 3.080420000 & 3.818590000\end{array}$


Table S15: Coordinates and energy of "Enolate-bound to $\gamma-\mathrm{Mo}_{2} \mathrm{~N}$ ", (Element Z, X, Y, Z)

\begin{tabular}{|c|c|c|c|c|c|c|c|}
\hline \multicolumn{8}{|c|}{$-918.965 \mathrm{eV}$} \\
\hline \multicolumn{8}{|c|}{$42 \quad 4.6760200$} \\
\hline 42 & 4.855020000 & 2.961810000 & 3.272320000 & 42 & 2.565820000 & 0.717150000 & 7.395340000 \\
\hline 42 & 6.710860000 & 0.993250000 & 3.205890000 & 42 & 2.790610000 & 3.021090000 & 5.454420000 \\
\hline 42 & 6.842330000 & 2.958920000 & 1.299130000 & 42 & 0.646740000 & 5.157730000 & 5.476410000 \\
\hline 42 & 8.909290000 & 0.847830000 & 1.252770000 & 42 & 0.611110000 & 6.975120000 & 7.467160000 \\
\hline 42 & 13.028420000 & 1.060690000 & 1.374780000 & 42 & 2.629240000 & 4.937120000 & 7.541560000 \\
\hline 42 & 8.816190000 & 2.967040000 & 3.283840000 & 42 & 2.613180000 & 6.917230000 & 5.651790000 \\
\hline 42 & 13.268180000 & 3.052620000 & 3.307500000 & 7 & 8.937530000 & 7.105660000 & 5.401710000 \\
\hline 42 & 10.918820000 & 0.947530000 & 3.287950000 & 7 & 13.102070000 & 5.057620000 & 7.458250000 \\
\hline 42 & 11.013120000 & 2.975510000 & 1.371350000 & 7 & 13.879720000 & 7.137880000 & 5.401610000 \\
\hline 42 & 4.720490000 & 5.010500000 & 1.311830000 & 7 & 12.821670000 & 2.963200000 & 5.436680000 \\
\hline 42 & 4.743570000 & 6.977390000 & 3.564380000 & 7 & 13.249270000 & 0.899820000 & 7.432610000 \\
\hline 42 & 6.827600000 & 4.993280000 & 3.346420000 & 7 & 4.930080000 & 2.912170000 & 5.415830000 \\
\hline 42 & 6.856010000 & 7.130870000 & 1.205430000 & 7 & 10.995420000 & 3.013070000 & 30000 \\
\hline 42 & 8.914530000 & 5.026240000 & 1.331980000 & 7 & 14.006210000 & 5.102970000 & 3.424800000 \\
\hline 42 & 13.056540000 & 4.937310000 & 1.453020000 & 7 & 6.808760000 & 7.106450000 & 3.365930000 \\
\hline 42 & 8.875720000 & 6.925380000 & 3.172440000 & 7 & 4.816450000 & 7.104950000 & 1.380670000 \\
\hline 42 & 13.022920000 & 7.009610000 & 3.528880000 & 7 & 13.114130000 & 60000 & 40000 \\
\hline 42 & 11.049110000 & 5.049880000 & 3.281090000 & 7 & 6.859680000 & 0.878520000 & 5.408090000 \\
\hline 42 & 11.004070000 & 0000 & 1.2 & 7 & 13.9 & 000 & 0000 \\
\hline 42 & 4.763010000 & 80000 & 000 & 7 & 13.0 & 000 & 000 \\
\hline 42 & 4.761490000 & 2.885080000 & 0000 & 7 & 0000 & 40000 & 1.237590000 \\
\hline 42 & 6.805030000 & 0.856420000 & 000 & 7 & 000 & 000 & 3.294200000 \\
\hline 42 & 6.977850000 & 3.043560000 & 5.427 & 7 & 0.447030000 & 7.144750000 & 5.603730000 \\
\hline 42 & 8.957790000 & 0.937670000 & 5.527690000 & 7 & 0.449000000 & 4.911220000 & 7.495910000 \\
\hline 42 & 12.910440000 & 0.719230000 & 0000 & 7 & 0000 & 0000 & 5.391740000 \\
\hline 42 & 8.869630000 & 2.942420000 & & 7 & & & 0000 \\
\hline 42 & 12.98 & 2.98 & & 7 & 00 & 7.1 & 000 \\
\hline 42 & 11.04 & 0000 & 7.4 & 7 & 0.44 & 5.02 & 10000 \\
\hline 42 & 10.677130000 & 3.014170000 & 0000 & 7 & 70000 & 0.762070000 & 7.336510000 \\
\hline 42 & 4.794580000 & 5.027410000 & 0000 & 7 & 2.684290000 & 0.847970000 & 5.302240000 \\
\hline 42 & 4.715640000 & 7.061670000 & 70000 & 7 & 10.911220000 & 2.995920000 & 7.436240000 \\
\hline 42 & 6.812200000 & 4.993690000 & 7.566720000 & 7 & 8.794890000 & 0.840870000 & 3.355160000 \\
\hline 42 & 6.771970000 & 7.035480000 & 5.536 & 7 & 10.983740000 & 7.153430000 & 3.340830000 \\
\hline 42 & 8.855550000 & 5.026210000 & & 7 & & & 3.270190000 \\
\hline 42 & 12.968 & 5.1 & & 1 & 15. & & 0000 \\
\hline 42 & 8.9330 & 0000 & 7.4 & 1 & 18.7 & 3.4 & 10000 \\
\hline 42 & 13.027260000 & 7.123560000 & 7.332880000 & 1 & 19.336970000 & 3.996030000 & 3.930290000 \\
\hline 42 & 10.933100000 & 5.108800000 & 7.50 & 1 & 17.460000000 & 2.597720000 & 5.052700000 \\
\hline 42 & 11.014640000 & 7.119370000 & 5.489590000 & 1 & 16.326040000 & 3.223480000 & 2.239960000 \\
\hline 42 & 0.591450000 & 1.126840000 & 1.255520000 & 1 & 19.908770000 & 1.595250000 & 4.520160000 \\
\hline 42 & 0.629250000 & 2.964470000 & 3.226190000 & 1 & 19.378480000 & 1.089180000 & 2.903910000 \\
\hline 42 & 2.670460000 & 1.024670000 & 3.170950000 & 1 & 20.785490000 & 2.169640000 & 3.083260000 \\
\hline 42 & 2.624040000 & 3.059860000 & 1.209940000 & 6 & 18.853030000 & 3.152680000 & 3.402580000 \\
\hline 42 & 0.615420000 & 4.922290000 & & 6 & 17.504030000 & 2.892290000 & 3.997910000 \\
\hline 42 & & & & 6 & 19.785950000 & 1.933520000 & 3.479480000 \\
\hline 42 & 2.647010000 & 4.961390000 & 3.369420000 & 6 & 16.343830000 & 2.948830000 & 3.307090000 \\
\hline 42 & 2.694740000 & 7.195570000 & 1.385590000 & 8 & 15.155640000 & 2.638330000 & 3.851050000 \\
\hline
\end{tabular}


Table S16: Coordinates and energy of "Enol-bound to $\gamma-\mathrm{Mo}_{2} \mathrm{~N}$ ", (Element Z, X, Y, Z)

$-918.603 \mathrm{eV}$

$42 \quad 4.683130000$

$42 \quad 4.868420000$

$42 \quad 6.771630000$

$42 \quad 6.867300000$

$42 \quad 8.951950000$

$42 \quad 13.016670000$

$42 \quad 8.755280000$

$42 \quad 13.047230000$

$42 \quad 11.149240000$

$42 \quad 10.996230000$

$42 \quad 4.721670000$

$42 \quad 4.739650000$

$42 \quad 6.787730000$

$42 \quad 6.865670000$

$42 \quad 8.939060000$

$42 \quad 12.993930000$

$42 \quad 8.852350000$

$42 \quad 12.978250000$

$\begin{array}{ll}42 & 10.949860000\end{array}$

$42 \quad 10.958770000$

$42 \quad 4.765030000$

$42 \quad 4.759760000$

$42 \quad 6.819890000$

$42 \quad 6.965600000$

$42 \quad 8.973590000$

$\begin{array}{ll}42 & 12.945460000\end{array}$

$42 \quad 8.869540000$

$42 \quad 12.993830000$

$42 \quad 10.984080000$

$42 \quad 10.722280000$

$42 \quad 4.791340000$

$42 \quad 4.711770000$

$\begin{array}{ll}42 & 6.779990000\end{array}$

$42 \quad 6.769900000$

$42 \quad 8.881460000$

$42 \quad 12.932530000$

$42 \quad 8.930330000$

$42 \quad 13.021900000$

$42 \quad 10.995430000$

$42 \quad 10.991190000$

$42 \quad 0.591450000$

$42 \quad 0.629250000$

$42 \quad 2.670460000$

$42 \quad 2.624040000$

$42 \quad 0.615420000$

$42 \quad 0.609530000$

$42 \quad 2.647010000$

$42 \quad 2.694740000$

$42 \quad 0.623110000$
0.893620000

2.933770000

0.993480000

2.944950000

0.851990000

0.979700000

3.038580000

2.912720000

0.883480000

2.990800000

5.020520000

6.986230000

4.988500000

7.149050000

5.029530000

4.875370000

6.889380000

7.122260000

5.024550000

7.116140000

0.710410000

2.864650000

0.845030000

3.019560000

0.926380000

0.706280000

2.923720000

2.941350000

0.845450000

2.978580000

5.011520000

7.070910000

4.970000000

7.005560000

5.011020000

5.186340000

7.074720000

7.106090000

5.047100000

7.089110000

1.126840000

2.964470000

1.024670000

3.059860000

4.922290000

7.086800000

4.961390000

7.195570000

0.539070000
1.288680000

3.244530000

3.205770000

1.260480000

1.273690000

1.357830000

3.288090000

3.233600000

3.312460000

1.342710000

1.318280000

3.568490000

3.313100000

1.187580000

1.283690000

1.354020000

3.152450000

3.563740000

3.318750000

1.226130000

5.470500000

7.509080000

7.499370000

5.424560000

5.485030000

5.410600000

7.532940000

7.616620000

7.462950000

5.393430000

5.494260000

7.405370000

7.585010000

5.529030000

5.460150000

5.416020000

7.475930000

7.342660000

7.447790000

5.492300000

1.255520000

3.226190000

3.170950000

1.209940000

1.394410000

3.686500000

3.369420000

1.385590000

5.313640000

$\begin{array}{cc}42 & 0.626920000 \\ 42 & 2.565820000 \\ 42 & 2.790610000 \\ 42 & 0.646740000 \\ 42 & 0.611110000 \\ 42 & 2.629240000 \\ 42 & 2.613180000 \\ 7 & 8.914580000 \\ 7 & 13.238540000 \\ 7 & 13.903890000 \\ 7 & 12.878490000 \\ 7 & 13.224380000 \\ 7 & 4.920640000 \\ 7 & 10.909250000 \\ 7 & 13.201160000 \\ 7 & 6.800310000 \\ 7 & 4.823030000 \\ 7 & 13.071820000 \\ 7 & 6.864520000 \\ 7 & 13.965940000 \\ 7 & 13.410430000 \\ 7 & 0.573670000 \\ 7 & 0.434740000 \\ 7 & 0.447030000 \\ 7 & 0.449000000 \\ 7 & 0.708950000 \\ 7 & 2.658680000 \\ 7 & 0.616260000 \\ 7 & 0.448360000 \\ 7 & 0.373870000 \\ 7 & 2.684290000 \\ 7 & 10.922960000 \\ 7 & 8.949060000 \\ 7 & 10.937020000 \\ 7 & 2.658730000 \\ 1 & 15.531570000 \\ 1 & 18.788230000 \\ 1 & 16.376310000 \\ 1 & 17.848140000 \\ 1 & 19.486310000 \\ 1 & 20.304420000 \\ 1 & 20.985980000 \\ 1 & 19.654890000 \\ 6 & 19.028070000 \\ 6 & 17.763080000 \\ 6 & 20.052340000 \\ 6 & 16.569000000 \\ 8 & 15.393370000 \\ & \end{array}$

2.825120000

7.522320000

0.717150000

3.021090000

5.157730000

6.975120000

4.937120000

6.917230000

7.087330000

5.024540000

7.094180000

2.947380000

0.866370000

2.895750000

2.944790000

5.031630000

7.114540000

7.112330000

7.087680000

0.857720000

2.935810000

0.847480000

3.032140000

0.886920000

7.144750000

4.911220000

2.940440000

7.113210000

7.186680000

5.024660000

0.762070000

0.847970000

2.946220000

0.889120000

7.133470000

2.993030000

2.578910000

3.248080000

3.565800000

2.239100000

1.861430000

3.397690000

3.825180000

4.788430000

2.853830000

2.698620000

3.768810000

3.116870000

3.047230000
7.395340000

5.454420000

5.476410000

7.467160000

7.541560000

5.651790000

5.385760000

7.430710000

5.407700000

5.407170000

7.427980000

5.402580000

3.364510000

3.388760000

3.353700000

1.379490000

1.340410000

5.389150000

1.305320000

3.390150000

1.237590000

3.294200000

5.603730000

7.495910000

5.391740000

3.477920000

1.460770000

3.430810000

7.336510000

5.302240000

7.413400000

3.338140000

3.334500000

3.270190000

4.748930000

1.797870000

2.190410000

4.584090000

2.639600000

4.491580000

2.906210000

3.598060000

2.799140000

3.591070000

3.486130000

3.165150000
3.899760000 
Table S17: Coordinates and energy of "CrotylAlcohol Alone", (Element Z, X, Y, Z)

$\begin{array}{llll}-71.633 \mathrm{eV} & & & \\ 1 & 16.351460000 & 3.325170000 & 3.782960000 \\ 1 & 21.310430000 & 3.772200000 & 1.944900000 \\ 1 & 21.088300000 & 4.627720000 & 3.474710000 \\ 1 & 20.982750000 & 2.848510000 & 3.437660000 \\ 1 & 15.384340000 & 2.032580000 & 2.220870000 \\ 1 & 16.691840000 & 4.090710000 & 2.201040000 \\ 1 & 18.623430000 & 2.129810000 & 3.632280000\end{array}$

Table S18: Coordinates and energy of "Crotonaldehyde Alone", (Element Z, X, Y, Z)

$-64.081 \mathrm{eV}$

$\begin{array}{llllllll}1 & 19.474500000 & 4.510240000 & 3.588960000 & 6 & 19.514120000 & 3.746990000 & 2.799690000 \\ 1 & 17.162440000 & 4.442800000 & 3.760080000 & 6 & 18.346820000 & 3.221750000 & 2.384300000 \\ 1 & 18.300640000 & 2.455000000 & 1.604220000 & 6 & 20.865090000 & 3.387030000 & 2.286520000 \\ 1 & 20.814310000 & 2.642800000 & 1.479840000 & 6 & 17.072960000 & 3.656360000 & 2.963750000 \\ 1 & 21.391570000 & 4.280130000 & 1.910040000 & 8 & 15.975890000 & 3.222020000 & 2.631140000 \\ 1 & 21.496410000 & 2.983980000 & 3.096960000 & & & & \end{array}$

Table S19: Coordinates and energy of "Enol Alone", (Element Z, X, Y, Z)

$-71.801 \mathrm{eV}$

$\begin{array}{llllllll}1 & 19.229200000 & 4.928210000 & 3.749310000 & 1 & 18.983570000 & 4.811810000 & 2.006620000 \\ 1 & 20.769540000 & 3.031110000 & 1.870890000 & 6 & 19.253950000 & 4.222010000 & 2.899000000 \\ 1 & 21.404910000 & 4.510170000 & 2.636370000 & 6 & 18.235320000 & 3.143030000 & 3.118010000 \\ 1 & 20.983480000 & 3.099690000 & 3.631050000 & 6 & 20.683960000 & 3.686920000 & 2.749500000 \\ 1 & 15.567920000 & 1.973130000 & 1.880580000 & 6 & 17.170590000 & 2.953230000 & 2.331990000 \\ 1 & 16.969220000 & 3.587980000 & 1.461770000 & 8 & 16.246600000 & 1.955850000 & 2.575590000 \\ 1 & 18.374080000 & 2.472870000 & 3.974030000 & & & & \end{array}$

Table S20: Coordinates and energy of "Butanal Alone", (Element Z, X, Y, Z)

$\begin{array}{llll}-72.120 \mathrm{eV} & & \\ 1 & 16.760250000 & 4.253920000 & 2.631900000 \\ 1 & 18.484650000 & 1.661930000 & 2.619020000 \\ 1 & 19.337660000 & 4.634000000 & 2.743760000 \\ 1 & 21.690700000 & 3.752240000 & 2.791600000 \\ 1 & 20.796590000 & 3.187460000 & 4.220760000 \\ 1 & 21.079960000 & 2.084530000 & 2.857050000 \\ 1 & 19.626210000 & 3.546270000 & 1.384010000\end{array}$

$\begin{array}{lll}18.939170000 & 4.735790000 & 2.026280000 \\ 19.265020000 & 3.869490000 & 2.616880000 \\ 18.332650000 & 3.012370000 & 3.050050000 \\ 20.733120000 & 3.765530000 & 2.884580000 \\ 16.869530000 & 3.186460000 & 2.813640000 \\ 16.355390000 & 2.009080000 & 2.156920000\end{array}$

